\title{
COMPACT TWO-SIDED KRYLOV METHODS FOR NONLINEAR EIGENVALUE PROBLEMS
}

\author{
PIETER LIETAERT*, KARL MEERBERGEN*, AND FRANÇOISE TISSEUR ${ }^{\dagger}$
}

\begin{abstract}
We describe a generalization of the compact rational Krylov (CORK) methods for polynomial and rational eigenvalue problems that usually but not necessarily come from polynomial or rational approximations of genuinely nonlinear eigenvalue problems. CORK is a family of one-sided methods that reformulates the polynomial or rational eigenproblem as a generalized eigenvalue problem. By exploiting the Kronecker structure of the associated pencil, it constructs a right Krylov subspace in compact form and thereby avoids the high memory and orthogonalization costs that are usually associated with linearizations of high degree matrix polynomials. CORK approximates eigenvalues and their corresponding right eigenvectors but is not suitable in its current form for the computation of left eigenvectors. Our generalization of the CORK method is based on a class of Kronecker structured pencils that include as special cases the CORK pencils, the transposes of CORK pencils, and the symmetrically structured linearizations by Robol, Vandebril and Van Dooren, 2016. This class of structured pencils facilitates the development of a general framework for the computation of both right and left-sided Krylov subspaces in compact form, and hence allows the development of two-sided compact rational Krylov methods for nonlinear eigenvalue problems. The latter are particularly efficient when the standard inner product is replaced by a cheaper to compute quasi inner product. We show experimentally that convergence results similar to CORK can be obtained for a certain quasi inner product.
\end{abstract}

Key words. nonlinear eigensolver, rational Krylov, nonlinear eigenvalue problem, two-sided Krylov method

AMS subject classifications. 65F15, 65F25, 15A18, 15A22, 41A20, 47J10

1. Introduction. We consider the computation of selected eigenvalues of the nonlinear eigenvalue problem and their associated right and left eigenvectors, which, for a given matrix-valued function $A: \mathbb{C} \rightarrow \mathbb{C}^{n \times n}$ consists of finding triplets $(\lambda, x, y)$ with $\lambda \in \mathbb{C}$ and nonzero vectors $x, y \in \mathbb{C}^{n}$ such that

$$
A(\lambda) x=0, \quad y^{*} A(\lambda)=0 .
$$

Applications are sensitivity analysis of eigenvalues (see [?], [?] and references therein) and eigenvalue optimization [?], [?].

Krylov methods are commonly used for finding eigenvalues of a matrix. The Arnoldi method [?] and the rational Krylov method [?] are one-sided methods, i.e., they build a Krylov space with the matrix. Two-sided methods build two Krylov spaces: one for the matrix and one for its transpose. Two-sided methods are less popular than one-sided methods because of the additional cost for building the left Krylov basis and possible loss of accuracy due to a potentially unstable biorthogonalization process. However, the computation of both right and left eigenvectors requires both Krylov spaces. The prototype two-sided Krylov method is the Lanczos method [?], [?]. The two-sided Arnoldi method builds two Arnoldi processes, one for the right Krylov space and one for the left Krylov space and uses oblique projections of the matrix on these spaces [?], [?].

When the coefficients of $A(\lambda)$ are polynomials in $\lambda, A(\lambda)$ is called a matrix polynomial. The eigenvalue problem is then called the polynomial eigenvalue problem. Its properties and solution are pretty well understood. Most numerical methods for computing a selection of eigenvalues strongly rely on the concept of linearization, that is, a linear pencil of larger size than $A$, that has exactly the same eigenvalues as the matrix polynomial. This generalized eigenvalue problem is then solved by an eigensolver for linear eigenvalue problems. Linearizations can also be used for solving nonlinear eigenvalue problems which are not polynomial, by first approximating the nonlinear functions by polynomials or even rationals. The matrix-valued function from the nonlinear

\footnotetext{
*Department of Computer Science, KU Leuven, University of Leuven, 3001 Heverlee, Belgium. Supported by KU Leuven Research Council Grant OT/14/074 and BELSPO Grant IAP VII/19.

${ }^{\dagger}$ School of Mathematics, The University of Manchester, Manchester, M13 9PL, UK. Supported by a Royal Society-Wolfson Research Merit Award.
} 
eigenvalue problem can, in general, be written as

$$
A(s)=\sum_{j=0}^{m} C_{j} f_{j}(s),
$$

where $C_{j} \in \mathbb{C}^{n \times n}$ are constant matrix factors and $f_{j}(s)$ are nonlinear scalar functions. In applications from physics and engineering such problems often have small $m$, e.g., three or four, which makes the approximation by a matrix polynomial or rational matrix function a little less complicated, since one just has to approximate the functions $f_{j}(s)$ by polynomials or rationals [?], [?], [?].

In this paper, we focus on polynomial or rational approximation $P(s)$ of the matrix-valued function $A(s)$ in (??). We consider rational Krylov methods for linearizations of $P(\lambda)$, which, in our context, is a pencil of order $n d$, where $d$ is the degree of the polynomial. The two orthogonal Arnoldi method (TOAR) [?], the compact rational Krylov method (CORK) [?], and related methods [?], [?], [?] all exploit the structure in the linearization so that the memory cost and the cost of the Gram-Schmidt orthogonalization process are reduced by almost a factor $d$ compared to applying a standard Krylov method directly to the linearization. Linearizations with compact representation of the Krylov vectors exist for many polynomial and rational bases. Most common is the companion linearization for matrix polynomials in monomial basis [?], [?]. Linearizations have been introduced and used for solving eigenvalue problems expressed in Newton and rational Newton polynomials [?], [?], Chebyshev polynomials [?], Lagrange polynomials [?], [?], rational monomials [?], [?], and the spectral discretization, which is also a rational approximation [?].

Two-sided methods already exist for the infinite Arnoldi method [?] and the delay Arnoldi method [?]. In this paper, we present a more generic approach that can be used for matrix polynomials or rational matrix-valued functions $P(s)$ that are expressed in polynomial or rational bases satisfying a linear relation. Both TOAR and CORK are designed for a compact representation of the right Krylov vectors $\mathbf{v}$ of the form $\mathbf{v}=(I \otimes Q) \mathbf{u}$, where $Q$ has orthonormal columns and $\mathbf{u}$ has much smaller dimension than $\mathbf{v}$. For the left Krylov vectors, however, the compact structure for the linearizations employed by CORK is, at first sight, completely lost. We show that the left Krylov subspace for CORK, and the right and the left Krylov subspaces for the symmetrically structured linearizations in [?] can also be represented in some compact form, but the latter is more expensive to exploit than for the one-sided CORK method. We show that the cost can be greatly reduced by employing special inner products in the Krylov methods. Due to the potential breakdown or loss of numerical stability of the two-sided Lanczos method, we use instead the two-sided Arnoldi method, i.e., we build two Krylov spaces, one for the matrix pencil of the linearization and one for its transpose. The eigenvalues and right and left eigenvectors are obtained from the two-sided projection of the pencil. Note that the cost for explicitly computing the oblique projection of the pencil on the left and right Krylov spaces does not increase the computational cost dramatically, at least for moderate size Krylov spaces.

The outline of the paper is as follows. In Section ??, we present the general framework of matrix polynomials using two types of linearizations: the CORK linearization [?] and symmetrically structured linearizations [?]. In Section ??, we introduce the compact form of the iteration vectors and present the algorithm for building the Krylov spaces using this form. We also introduce an alternative inner product to reduce the computational cost. Section 4 illustrates the methods by numerical examples, where we compare accuracy and memory cost for various alternatives.

We use lowercase Roman and Greek letters for numbers (e.g. $f, \phi)$, uppercase Greek letters and lowercase Roman letters to indicate vectors (e.g. $\Phi$ or $v$ ), capital letters for matrices (e.g. $A$ ), boldface lower and upper case letters for block vectors and matrices (e.g. $\mathbf{v}, \mathbf{A}$ ) and blackboard boldface letters for matrices containing different block structures (e.g. $\mathbb{A})$. We denote by $e_{j}$ the unit vector with zero entries everywhere except in position $j$, where it is one. We will often use the $n \times(n-1)$ matrix

$$
\check{I}_{n}=\left[\begin{array}{c}
0 \\
I_{n-1}
\end{array}\right],
$$


which corresponds to the $n \times n$ identity matrix $I_{n}$ with its first column removed. When partitioning a vector $\mathbf{v} \in \mathbb{C}^{n d}$ in $d$ blocks of length $n, v^{[i]}$ indicates the $i$ th block.

2. Matrix polynomials, rational functions and their linearizations. Assume the matrix-valued function in (??) is approximated by a matrix polynomial or rational matrix function of the form

$$
P(s)=P_{0} \xi_{0}(s)+P_{1} \xi_{1}(s)+\cdots+P_{d} \xi_{d}(s),
$$

where $P_{j} \in \mathbb{C}^{n \times n}$ are constant coefficient matrices and $\xi_{j}(s)$ are rational functions or polynomials. This approximation can, for example, be obtained using one of the techniques described in [?, Section 6]. We concentrate on basis functions $\xi_{j}(s)$ that satisfy a linear recurrence relation. These include:

(a) shifted and scaled monomials $\xi_{j}(s)=(s-\sigma)^{j} / \prod_{i=0}^{j} \beta_{i}$, which satisfy the linear relation

$$
\xi_{0}(s)=\frac{1}{\beta_{0}}, \quad \xi_{j}(s)=\frac{s-\sigma}{\beta_{j}} \xi_{j-1}(s), \quad j \geq 1,
$$

for some nonzero scaling factors $\beta_{j}$ and shift $\sigma$;

(b) orthogonal polynomials, which satisfy a three-term recurrence

$$
\xi_{-1}(s)=0, \quad \xi_{0}(s)=1, \quad s \xi_{j}(s)=\alpha_{j} \xi_{j+1}(s)+\beta_{j} \xi_{j}(s)+\gamma_{j} \xi_{j-1}(s), \quad j \geq 0,
$$

(note that both the shifted and scaled monomials (??), and the Chebyshev polynomials

$$
T_{0}(s)=1, \quad T_{1}(s)=s, \quad T_{j+1}(s)=2 s T_{j}(s)-T_{j-1}(s), \quad j \geq 1,
$$

are special cases of (??));

(c) rational Newton basis functions, which are defined by the recursion

$$
\xi_{0}(s)=\frac{1}{\beta_{0}}, \quad \xi_{j}(s)=\frac{s-\sigma_{j}}{\beta_{j}\left(1-s / \zeta_{j}\right)} \xi_{j-1}(s), \quad j \geq 1,
$$

for some scaling factors $\beta_{0}, \ldots, \beta_{j}$, interpolation nodes $\sigma_{1}, \ldots, \sigma_{j}$ and poles $\zeta_{1}, \ldots, \zeta_{j}$ (note that scaled Newton polynomials, which are defined by the linear relation

$$
\xi_{0}(s)=\frac{1}{\beta_{0}}, \quad \xi_{j}(s)=\frac{s-\sigma_{j}}{\beta_{j}} \xi_{j-1}(s), \quad j \geq 1,
$$

are a special case of (??) corresponding to choosing poles at infinity);

(d) scaled Lagrange polynomials with distinct nodes $\sigma_{0}, \ldots, \sigma_{d}$ and scaling factors $\beta_{0}, \ldots, \beta_{d}$,

$$
\xi_{j}(s)=\frac{1}{\beta_{j}} \prod_{\substack{i=0 \\ i \neq j}}^{d}\left(s-\sigma_{i}\right), \quad 0 \leq j \leq d,
$$

which satisfy the linear relation

$$
\beta_{j}\left(s-\sigma_{j}\right) \xi_{j}(s)=\beta_{j-1}\left(s-\sigma_{j-1}\right) \xi_{j-1}(s), \quad j>0 .
$$

The linear recurrence relation between the basis functions can be rewritten in matrix form as

$$
M_{d} \Xi_{d}(s)=s N_{d} \Xi_{d}(s),
$$

where

$$
\Xi_{d}(s)=\left[\xi_{0}(s), \xi_{1}(s), \ldots, \xi_{d}(s)\right]^{T}
$$

and $M_{d}, N_{d} \in \mathbb{C}^{d \times(d+1)}$. The structure of $M_{d}$ and $N_{d}$ is provided in Table ?? for the standard bases (??)-(??). When (??) holds with $M_{d}-s N_{d}$ of full row rank, then $M_{d}-s N_{d}$ is said to be a 
TABLE 2.1

Linear dual bases $M_{d}-s N_{d}$ for the standard polynomial bases $\Xi_{d}(s)=\left[\xi_{0}(s), \xi_{1}(s), \ldots, \xi_{d}(s)\right]^{T}$ in (??)-(??).

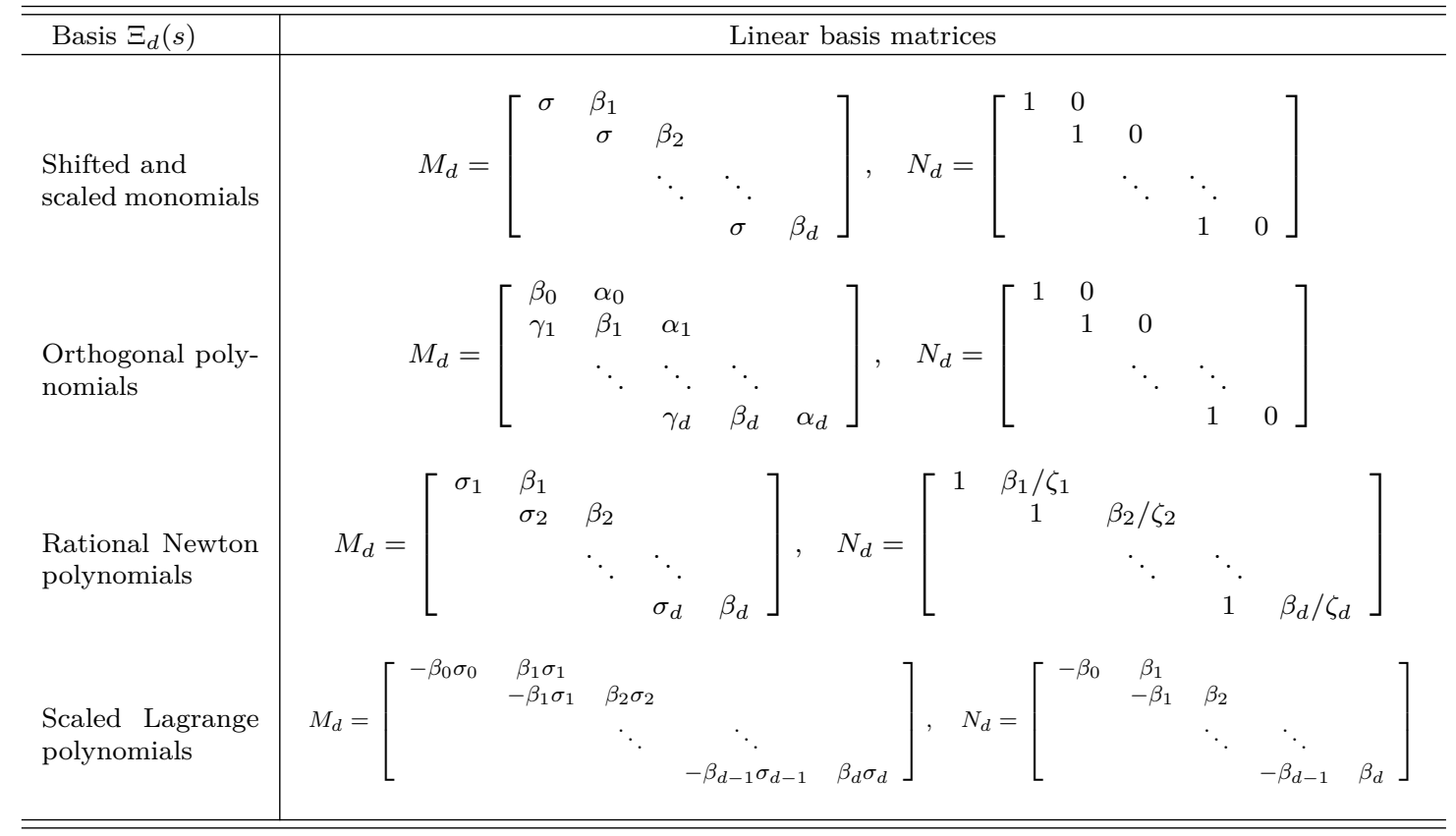

full row-rank linear dual basis to $\Xi_{d}(s)$ [?], [?, Definition 12]. Note that in the special case $d=0$, we consider the corresponding dual linear basis to be empty.

Using the linear relation between $\xi_{d}(s)$ and $\xi_{d-1}(s)$, the matrix polynomial or rational matrix function $P(s)$ in (??) is rewritten in the form

$$
g(s) P(s)=\sum_{j=0}^{d-1}\left(A_{j}-s B_{j}\right) \xi_{j}(s),
$$

for some function $g(s)$ and matrices $A_{j}, B_{j}$ that depend on the coefficient matrices $P_{j}$ of $P(s)$ in (??) - see for example [?], [?, Table 6.6]. Note that $g(s)=1$ for polynomial bases but $g(s)=$ $\left(1-s / \zeta_{d}\right)$ for the rational Newton basis.

Define

$$
\mathbf{A}-s \mathbf{B}=\left[\begin{array}{llll}
A_{0}-s B_{0} & A_{1}-s B_{1} & \cdots & A_{d-1}-s B_{d-1}
\end{array}\right] \in \mathbb{C}^{n \times n d},
$$

and rewrite (??) as

$$
g(s) P(s)=(\mathbf{A}-s \mathbf{B})\left(\Xi_{d-1}(s) \otimes I_{n}\right) .
$$

The pencil

$$
\mathbb{A}-s \mathbb{B}=\left[\begin{array}{c}
\mathbf{A}-s \mathbf{B} \\
\left(M_{d-1}-s N_{d-1}\right) \otimes I_{n}
\end{array}\right] \in \mathbb{C}^{n d \times n d}
$$

is referred to as the CORK pencil [?]. It is a linearization of $P(s)$ when the latter is a matrix polynomial. The CORK pencil satisfies two one-sided factorizations as the next lemma shows.

LEMMA 2.1. Let $\Xi_{d-1}(s)=\left[\xi_{0}(s), \ldots, \xi_{d-1}(s)\right]^{T}$ be a family of basis functions satisfying the linear relation $M_{d-1} \Xi_{d-1}(s)=s N_{d-1} \Xi_{d-1}(s)$. For every $s \in \mathbb{C}$ such that the $d-1$ last columns of $M_{d-1}-s N_{d-1}$ are linearly independent and $\xi_{0}(s) \neq 0$, the pencil $\mathbb{A}-s \mathbb{B}$ in (??) and $g(s) P(s)$ in (??) satisfy the 
(i) right-sided factorization

$$
(\mathbb{A}-s \mathbb{B}) G(s)=e_{1} \otimes g(s) P(s),
$$

where $G(s)=\Xi_{d-1}(s) \otimes I_{n}$ and $e_{1}$ is the first column of the $d \times d$ identity matrix, and the (ii) left-sided factorization

$$
H(s)(\mathbb{A}-s \mathbb{B})=e_{1}^{T} \otimes g(s) P(s),
$$

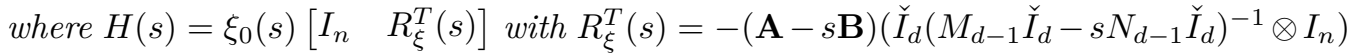
and $\check{I}_{d}$ as in (??).

Proof. Statement (i) follows from $\left(M_{d-1}-s N_{d-1}\right) \Xi_{d-1}(s)=0$ and (??). See also [?].

For statement (ii), we start by rewriting $\left(M_{d-1}-s N_{d-1}\right) \Xi_{d-1}=0$ as

$$
\xi_{0}(s)\left(M_{d-1}-s N_{d-1}\right) e_{1}=-\left(M_{d-1}-s N_{d-1}\right) \check{I}_{d} \check{I}_{d}^{T} \Xi_{d-1}(s) .
$$

Since $s$ is such that $\left(M_{d-1}-s N_{d-1}\right) \check{I}_{d}$ is nonsingular and $\xi_{0}(s) \neq 0$, we can write (??) as

$$
\left(M_{d-1} \check{I}_{d}-s N_{d-1} \check{I}_{d}\right)^{-1}\left(M_{d-1}-s N_{d-1}\right) e_{1}=-\check{I}_{d}^{T} \Xi_{d-1}(s) / \xi_{0}(s) .
$$

Hence, $\left(M_{d-1} \check{I}_{d}-s N_{d-1} \check{I}_{d}\right)^{-1}\left(M_{d-1}-s N_{d-1}\right)=\left[\begin{array}{ll}-\check{I}_{d}^{T} \Xi_{d-1}(s) / \xi_{0}(s) & I_{d-1}\end{array}\right]$, so that

$$
\xi_{0}(s)\left(I_{d}-\check{I}_{d}\left(M_{d-1} \check{I}_{d}-s N_{d-1} \check{I}_{d}\right)^{-1}\left(M_{d-1}-s N_{d-1}\right)\right)=e_{1}^{T} \otimes \Xi_{d-1}(s) .
$$

Now, the left-hand side of (??) can be rewritten as

$$
\xi_{0}(s)(\mathbf{A}-s \mathbf{B})-\xi_{0}(s)(\mathbf{A}-s \mathbf{B})\left(\check{I}_{d}\left(M_{d-1} \check{I}_{d}-s N_{d-1} \check{I}_{d}\right)^{-1}\left(M_{d-1}-s N_{d-1}\right) \otimes I_{n}\right) .
$$

From (??), after Kronecker product with $I_{n}$, we have that (??) equals

$$
(\mathbf{A}-s \mathbf{B})\left(e_{1}^{T} \otimes \Xi_{d-1}(s) \otimes I_{n}\right),
$$

which, by (??), equals $e_{1}^{T} \otimes g(s) P(s)$.

The next result describes the structure of the eigenvectors of $\mathbb{A}-s \mathbb{B}$. It is a direct consequence of [?, Thm. 3.1] applied to the one-sided factorizations (??)-(??).

THEOREM 2.2. Let $P(s)$ be the $n \times n$ matrix polynomial or rational matrix-valued function in (??), let $\mathbb{A}-s \mathbb{B}$ be the $n d \times$ nd pencil in (??), and let $\lambda \in \mathbb{C}$.

(i) If (??) holds at $\lambda$ with $\Xi_{d-1}(\lambda) \neq 0$ then $\Xi_{d-1}(\lambda) \otimes x$ is a right eigenvector of $\mathbb{A}-s \mathbb{B}$ with eigenvalue $\lambda$ if and only if $x$ is a right eigenvector of $P(s)$ with eigenvalue $\lambda$.

(ii) If (??) holds at $\lambda$ with $H(\lambda)$ of full rank then $\left[\frac{y}{R_{\xi}(\lambda)} y\right]$ is a left eigenvector of $\mathbb{A}-s \mathbb{B}$ with eigenvalue $\lambda$ if and only if $y$ is a left eigenvector of $P(s)$ with eigenvalue $\lambda$.

The conditions $\left(M_{d-1}-s N_{d-1}\right) \check{I}_{d}$ being nonsingular and $\xi_{0}(s) \neq 0$ are not too strict since we can permute the order of the entries in the basis $\Xi_{d-1}$ if needed. Note that for degree graded polynomial bases, $\xi_{0}(s)$ is a constant and therefore nonzero.

EXAMPLE 1. The degree d matrix polynomial expressed in the monomial basis as

$$
P(s)=P_{0}+s P_{1}+s^{2} P_{2}+\ldots+s^{d} P_{d},
$$

can be rewritten in the form (??) with $g(s)=1, A_{i}=P_{i}, B_{i}=0$ for $i=0, \ldots, d-2$ and $A_{d-1}=P_{d-1}, B_{d-1}=-P_{d}$. Then the pencil $\mathbb{A}-s \mathbb{B}$ in (??) has the form

$$
\mathbb{A}=\left[\begin{array}{cccc}
P_{0} & P_{1} & \cdots & P_{d-1} \\
0 & I_{n} & & \\
& \ddots & \ddots & \\
& & 0 & I_{n}
\end{array}\right], \quad \mathbb{B}=\left[\begin{array}{cccc}
0 & \cdots & 0 & -P_{d} \\
I_{n} & 0 & & \\
& \ddots & \ddots & \\
& & I_{n} & 0
\end{array}\right]
$$


which is close to the first companion form [?], [?].

From Theorem ??, the right and left eigenvectors of $\mathbb{A}-s \mathbb{B}$, with eigenvalue $\lambda$, have the form

$$
\mathbf{x}=\left[\begin{array}{c}
x \\
\lambda x \\
\lambda^{2} x \\
\vdots \\
\lambda^{d-1} x
\end{array}\right], \quad \mathbf{y}=\left[\begin{array}{c}
y \\
-\left(\sum_{i=1}^{d} \lambda^{i-1} P_{i}\right)^{*} y \\
-\left(\sum_{i=2}^{d} \lambda^{i-2} P_{i}\right)^{*} y \\
\vdots \\
-\left(P_{d-1}+\lambda P_{d}\right)^{*} y
\end{array}\right]
$$

The blocks of the right eigenvector are just scalar multiples of the right eigenvector $x$ of $P(s)$ whereas the structure of the left eigenvector is more complicated.

As we are interested in computing both right and left eigenvectors of $P(s)$, we also consider a class of symmetrically structured pencils associated with $P(s)$ whose right and left eigenvectors have a similar Kronecker product form. Assuming for simplicity that $d$ is odd we rewrite $P(s)$ in (??) as

$$
g(s) P(s)=\sum_{j=0}^{\frac{d-1}{2}}\left(A_{j}-s B_{j}\right) \phi_{j}(s) \psi_{j}(s),
$$

for some function $g(s)$ and matrices $A_{j}, B_{j}$ that depend on the coefficient matrices $P_{j}$ of $P(s)$ in (??) (note that these differ in general from $g(s)$ and $A_{j}, B_{j}$ in (??) but for the presentation of our general framework, it is convenient to not introduce new notation here). The $\phi_{j}(s), j=1, \ldots, d_{\phi}$ and the $\psi_{j}(s), j=0, \ldots, d_{\psi}$ with $d_{\phi}=d_{\psi}=\frac{d-1}{2}$ are two families of basis functions, each of them satisfying a linear relation such that if

$$
\Phi_{d_{\phi}}(s)=\left[\phi_{0}(s), \ldots, \phi_{d_{\phi}}(s)\right]^{T}, \quad \Psi_{d_{\psi}}(s)=\left[\psi_{0}(s), \ldots, \psi_{d_{\psi}}(s)\right]^{T}
$$

then

$$
\left(M_{d_{\phi}}-s N_{d_{\phi}}\right) \Phi_{d_{\phi}}(s)=0, \quad\left(M_{d_{\psi}}-s N_{d_{\psi}}\right) \Psi_{d_{\psi}}(s)=0,
$$

with $M_{d_{\phi}}, N_{d_{\phi}} \in \mathbb{C}^{d_{\phi} \times\left(d_{\phi}+1\right)}$ and $M_{d_{\psi}}, N_{d_{\psi}} \in \mathbb{C}^{d_{\psi} \times\left(d_{\psi}+1\right)}$. Now define the $n\left(d_{\psi}+1\right) \times n\left(d_{\phi}+1\right)=$ $n\left(\frac{d+1}{2}\right) \times n\left(\frac{d+1}{2}\right)$ pencil

$$
\mathbf{A}-s \mathbf{B}=\left[\begin{array}{llll}
A_{0}-s B_{0} & & & \\
& A_{1}-s B_{1} & & \\
& & \ddots & \\
& & & A_{\frac{d-1}{2}}-s B_{\frac{d-1}{2}}
\end{array}\right]
$$

and rewrite (??) as

$$
g(s) P(s)=\left(\Psi_{d_{\psi}}(s) \otimes I_{n}\right)^{T}(\mathbf{A}-s \mathbf{B})\left(\Phi_{d_{\phi}}(s) \otimes I_{n}\right) .
$$

When $P(s)$ is a matrix polynomial, both $M_{d_{\phi}}-s N_{d_{\phi}}$ and $M_{d_{\psi}}-s N_{d_{\psi}}$ are of full row-rank with $d_{\phi}=d_{\psi}$, and the elements in the polynomial basis $\Phi_{d_{\phi}}(s)$ (respectively, $\Psi_{d_{\psi}}(s)$ ) have no common divisor, Robol et al [?, Thm. 15] show that

$$
\mathbb{A}-s \mathbb{B}=\left[\begin{array}{cc}
\mathbf{A}-s \mathbf{B} & \left(M_{d_{\psi}}-s N_{d_{\psi}}\right)^{T} \otimes I_{n} \\
\left(M_{d_{\phi}}-s N_{d_{\phi}}\right) \otimes I_{n} & 0
\end{array}\right] \in \mathbb{C}^{n d \times n d}
$$

is a linearization of $P(s)$. The pencil is symmetrically structured if both $M_{d_{\phi}}-s N_{d_{\phi}}$ and $M_{d_{\psi}}-$ $s N_{d_{\psi}}$ have the same structure. This is, for example, the case if we use two polynomial bases $\Phi_{d_{\phi}}(s)$ and $\Psi_{d_{\psi}}(s)$ of the same type. Furthermore, if both bases are identical, i.e., $\Phi_{d_{\phi}}(s)=\Psi_{d_{\psi}}(s)$ and $\mathbf{A}-s \mathbf{B}$ is symmetric, then $\mathbb{A}-s \mathbb{B}$ is symmetric.

The rational matrix function or matrix polynomial $P(s)$ can be rewritten in the form (??) in several ways. We describe below a possibility for the standard basis functions (??)-(??). 
(a) If $P$ in (??) is expressed in shifted and scaled monomial basis, then, for the $\phi_{j}$ and $\psi_{j}$ in (??), we can choose the shifted and scaled monomials

$$
\begin{array}{ll}
\phi_{0}(s)=\frac{1}{\beta_{0}}, & \phi_{j}(s)=\frac{(s-\sigma)}{\beta_{2 j}} \phi_{j-1}(s), \quad j=0, \ldots(d-1) / 2, \\
\psi_{0}(s)=\frac{1}{\beta_{1}}, & \psi_{j}(s)=\frac{(s-\sigma)}{\beta_{2 j+1}} \psi_{j-1}(s), \quad j=0, \ldots,(d-1) / 2
\end{array}
$$

so that

$$
A_{j}=\beta_{2 j+1} P_{2 j}-\sigma P_{2 j+1}, \quad B_{j}=-P_{2 j+1}, \quad j=0, \ldots,(d-1) / 2
$$

and $g(s)=1$.

(b) For Chebyshev polynomials of the first or second kind, see [?].

(c) If the $\xi(s)$ are the scaled rational Newton basis functions in (??) then for $\left(1-\frac{s}{\xi_{1}}\right) P(s)$ (i.e., $g(s)=1-s / \xi_{1}$ ), we can choose

$$
\begin{aligned}
& \phi_{0}(s)=\frac{1}{\beta_{0}}, \quad \phi_{j}(s)=\frac{s-\sigma_{2 j-1}}{\beta_{2 j}\left(1-s / \zeta_{2 j}\right)} \phi_{j-1}, \quad j=1, \ldots,(d-1) / 2, \\
& \psi_{0}(s)=\frac{1}{\beta_{1}}, \quad \psi_{j}(s)=\frac{s-\sigma_{2 j}}{\beta_{2 j+1}\left(1-s / \zeta_{2 j+1}\right)} \psi_{j-1}, \quad j=1, \ldots,(d-1) / 2,
\end{aligned}
$$

so that

$$
A_{j}=\beta_{2 j+1} P_{2 j}-\sigma_{2 j+1} P_{2 j+1}, \quad B_{j}=\frac{\beta_{2 j+1}}{\xi_{2 j+1}} P_{2 j}-P_{2 j+1}, \quad j=0, \ldots,(d-1) / 2 .
$$

(e) If $P$ in (??) is expressed in the scaled Lagrange basis $\xi_{j}(s), j=0, \ldots, d$ with $\xi_{j}$ as in (??), then as suggested in [?], we can choose the scaled Lagrange basis functions

$$
\phi_{j}(s)=\frac{1}{\beta_{2 j}} \prod_{\substack{i=0 \\ i \neq j}}^{(d-1) / 2}\left(s-\sigma_{2 i}\right), \quad \psi_{j}(s)=\frac{1}{\beta_{2 j+1}} \prod_{\substack{i=0 \\ i \neq j}}^{(d-1) / 2}\left(s-\sigma_{2 i+1}\right), \quad j=0, \ldots,(d-1) / 2
$$

so that

$$
A_{j}=-\sigma_{2 j+1} \beta_{2 j+1} P_{2 j}-\sigma_{2 j} \beta_{2 j} P_{2 j+1}, \quad B_{j}=-\beta_{2 j+1} P_{2 j}-\beta_{2 j} P_{2 j+1}, \quad j=0, \ldots,(d-1) / 2 .
$$

Note that the expression for $P(s)$ in (??) is a particular case of (??) with $d_{\psi}=0, \Psi_{d_{\psi}}=[1]$, $d_{\phi}=d-1$ and $\mathbf{A}-s \mathbf{B}$ as in (??). The next result extends Lemma ?? to the more general expression for $P(s)$ in (??) and its associated pencil $\mathbb{A}-s \mathbb{B}$ in (??).

LEMma 2.3. Let $\Phi_{d_{\phi}}(s)$ and $\Psi_{d_{\psi}}(s)$ be the two families of basis functions in (??) satisfying the linear relations (??).

(i) Let $s \in \mathbb{C}$ be such that $\psi_{0}(s) \neq 0$ and the last $d_{\psi}$ columns of $M_{d_{\psi}}-s N_{d_{\psi}}$ are linearly independent. Then $P(s)$ in (??) and the matrix pencil $\mathbb{A}-s \mathbb{B}$ in (??) satisfy the right-sided factorization

$$
(\mathbb{A}-s \mathbb{B}) G(s)=e_{1} \otimes g(s) P(s),
$$

where

$$
G(s)=\psi_{0}(s)\left[\begin{array}{c}
I_{n\left(d_{\phi}+1\right)} \\
R_{\psi}(s)
\end{array}\right]\left(\Phi_{d_{\phi}}(s) \otimes I_{n}\right)
$$

with $R_{\psi}(s)=-\left(\left(\left(M_{d_{\psi}}-s N_{d_{\psi}}\right) \check{I}_{d_{\psi}+1}\right)^{-T} \check{I}_{d_{\psi}+1}^{T} \otimes I_{n}\right)(\mathbf{A}-s \mathbf{B}) \in \mathbb{C}^{n d_{\psi} \times n\left(d_{\phi}+1\right)}$. 
(ii) Let $s \in \mathbb{C}$ be such that $\phi_{0}(s) \neq 0$ and the last $d_{\phi}$ columns of $M_{d_{\phi}}-s N_{d_{\phi}}$ are linearly independent. Then $P(s)$ in (??) and the matrix pencil $\mathbb{A}-s \mathbb{B}$ in (??) satisfy the left-sided factorization

$$
H(s)(\mathbb{A}-s \mathbb{B})=e_{1}^{T} \otimes g(s) P(s),
$$

where

$$
H(s)=\phi_{0}(s)\left(\Psi_{d_{\psi}}(s)^{T} \otimes I_{n}\right)\left[I_{n\left(d_{\psi}+1\right)} \quad R_{\phi}^{T}(s)\right]
$$

with $R_{\phi}^{T}(s)=-(\mathbf{A}-s \mathbf{B})\left(\check{I}_{d_{\phi}+1}\left(\left(M_{d_{\phi}}-s N_{d_{\phi}}\right) \check{I}_{d_{\phi}+1}\right)^{-1} \otimes I_{n}\right) \in \mathbb{C}^{n\left(d_{\psi}+1\right) \times n d_{\phi}}$.

Proof. The proof that the last $n d_{\phi}$ rows of (??) and the last $n d_{\psi}$ columns of (??) are zero follows from (??). The proof that the first $n\left(d_{\psi}+1\right)$ rows of (??) and the first $n\left(d_{\phi}+1\right)$ columns of (??) are equal to $e_{1} \otimes g(s) P(s)$ and $e_{1}^{T} \otimes g(s) P(s)$, respectively, is similar to the proof of statement (ii) of Lemma ??.

The next result follows from [?, Thm. 3.1] and the two one-side factorizations in Lemma ??.

THEOREM 2.4. Let $P(\lambda)$ be the $n \times n$ matrix polynomial or rational matrix-valued function in (??), $\mathbb{A}-s \mathbb{B}$ be the $n d \times$ nd pencil in (??) and let $\lambda \in \mathbb{C}$.

(i) If (??) holds at $\lambda$ with $G(\lambda)$ of full rank, then

- $\left[\begin{array}{c}\Phi_{d_{\phi}}(\lambda) \otimes x \\ R_{\psi}(\lambda)\left(\Phi_{d_{\phi}}(\lambda) \otimes x\right)\end{array}\right]$ is a right eigenvector of $\mathbb{A}-s \mathbb{B}$ with eigenvalue $\lambda$ if and only if $x$ is a right eigenvector of $P(s)$ with eigenvalue $\lambda$.

- if $w \in \mathbb{C}^{\text {nd }}$ is a left eigenvector of $\mathbb{A}-s \mathbb{B}$ with eigenvalue $\lambda$ then $\left(e_{1}^{T} \otimes I_{n}\right) w$ is a left eigenvector of $P(s)$ with eigenvalue $\lambda$ provided that it is nonzero.

(ii) If (??) holds at $\lambda$ with $H(\lambda)$ of full rank, then

- If $z \in \mathbb{C}^{\text {nd }}$ is a right eigenvector of $\mathbb{A}-s \mathbb{B}$ with eigenvalue $\lambda$ then $\left(e_{1}^{T} \otimes I_{n}\right) z$ is a right eigenvector of $P$ with eigenvalue $\lambda$ provided that it is nonzero.

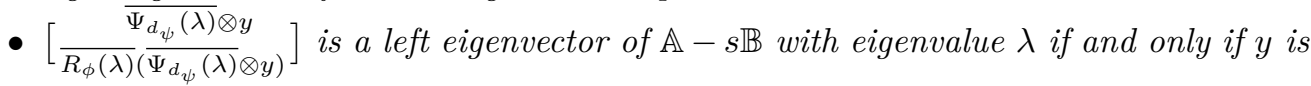
a left eigenvector of $P$ with eigenvalue $\lambda$.

Note that we can again use permutation matrices in order to relax the conditions on $\left(M_{d_{\phi}}-\right.$ $\left.s N_{d_{\phi}}\right) \check{I}_{d_{\phi}+1},\left(M_{d_{\psi}}-s N_{d_{\psi}}\right) \check{I}_{d_{\psi}+1}$ being nonsingular, and on $\phi_{0}(s)$ and $\psi_{0}(s)$ being nonzero.

EXAMPle 2. For matrix polynomials of odd degree expressed in the monomial basis (??), we select $\phi_{j}(s)=s^{j}, \psi_{j}(s)=s^{j}$ for $j=0, \ldots, \frac{d-1}{2}$, so that $g(s)=1, A_{j}=P_{2 j}, B_{j}=-P_{2 j+1}$ for $j=0, \ldots, \frac{d-1}{2}$. The $n d \times$ nd pencil (??) is, in this case, a permuted Fiedler linearization [?]:

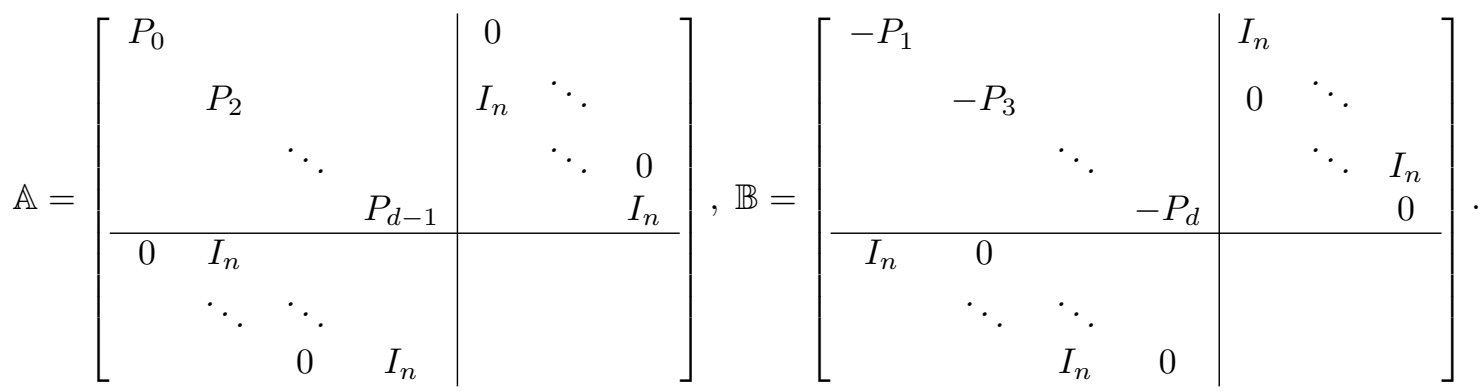

It follows from Theorem ?? that the left and right eigenvectors of $\mathbb{A}-s \mathbb{B}$ with eigenvalue $\lambda$ have 
the form

$$
\mathbf{x}=\left[\begin{array}{c}
x \\
\lambda x \\
\vdots \\
\lambda^{\frac{d-1}{2}} x \\
-\left(\sum_{i=2}^{d} \lambda^{i-1} P_{i}\right) x \\
-\left(\sum_{i=4}^{d} \lambda^{i-2} P_{i}\right) x \\
\vdots \\
-\left(\lambda^{\frac{d-1}{2}}\left(P_{d-1}+\lambda P_{d}\right)\right) x
\end{array}\right], \quad \mathbf{y}=\left[\begin{array}{c}
y \\
\bar{\lambda} y \\
\vdots \\
\bar{\lambda} \frac{d-1}{2} y \\
-\left(\sum_{i=2}^{d} \lambda^{i-1} P_{i}\right)^{*} y \\
-\left(\sum_{i=4}^{d} \lambda^{i-2} P_{i}\right)^{*} y \\
\vdots \\
-\left(\lambda^{\frac{d-1}{2}}\left(P_{d-1}+\lambda P_{d}\right)\right)^{*} y
\end{array}\right]
$$

where $x$ and $y$ are right and left eigenvectors for $P$ with eigenvalue $\lambda$.

In what follows, we consider our matrix valued function $P(s)$ to be in the form (??) with corresponding pencil (??), which includes the three special cases:

$\mathbf{L}_{1}$ : the CORK pencil for which

- $d_{\psi}=0, \Psi_{d_{\psi}}(s)=[1], d_{\phi}=d-1$ and $\Phi_{d_{\phi}}(s)=\Xi_{d-1}(s)$, with $\Xi_{d-1}(s)$ as in (??),

- $\mathbf{A}-s \mathbf{B}$ has the form (??) and $\left(M_{d_{\psi}}-s N_{d_{\psi}}\right)^{T} \otimes I_{n}$ is the empty matrix;

$\mathbf{L}_{2}$ : the transpose of the CORK pencil, i.e., $\mathbf{L}_{2}=\mathbf{L}_{1}^{T}$ for which

- $d_{\psi}=d-1, \Psi_{d_{\psi}}(s)=\Xi_{d-1}(s), d_{\phi}=0$ and $\Phi_{d_{\phi}}(s)=[1]$,

- $\mathbf{A}-s \mathbf{B}$ is the transpose of (??) and where $A_{i}, B_{i}, i=0, \ldots, d-1$ are also transposed, and $\left(M_{d_{\phi}}-s N_{d_{\phi}}\right) \otimes I_{n}$ is the empty matrix;

$\mathbf{L}_{S}:$ the symmetrically structured pencil (??) for which

- $d_{\psi}=d_{\phi}=(d-1) / 2$, and $\Psi_{d_{\psi}}(s)$ and $\Phi_{d_{\psi}}(s)$ are as in (??),

- $\mathbf{A}-s \mathbf{B}$ has the form (??).

3. Compact Krylov methods. The (one-sided) rational Krylov method for the $n d \times n d$ pencil $\mathbb{A}-s \mathbb{B}$, outlined in Algorithm ??, builds an orthonormal basis $\mathbf{V}_{k+1}=\left[\mathbf{v}_{1}, \ldots, \mathbf{v}_{k+1}\right] \in$ $\mathbb{C}^{n d \times(k+1)}$ for the (right) Krylov space

$$
\operatorname{span}\left\{\mathbf{V}_{k+1}\right\}=\operatorname{span}\left\{\mathbf{v}_{1},\left(\mathbb{A}-\sigma_{1} \mathbb{B}\right)^{-1} \mathbb{B} \mathbf{v}_{1}, \ldots,\left(\mathbb{A}-\sigma_{k} \mathbb{B}\right)^{-1} \mathbb{B} \mathbf{v}_{1}\right\},
$$

for $k$ distinct shifts $\sigma_{1}, \ldots, \sigma_{k}$ [?]. If all the shifts are equal, the method is equivalent to the shift-and-invert Arnoldi method, which builds the Krylov space

$$
\operatorname{span}\left\{\mathbf{v}_{1},\left(\mathbb{A}-\sigma_{1} \mathbb{B}\right)^{-1} \mathbb{B} \mathbf{v}_{1}, \ldots,\left(\left(\mathbb{A}-\sigma_{1} \mathbb{B}\right)^{-1} \mathbb{B}\right)^{k} \mathbf{v}_{1}\right\} .
$$

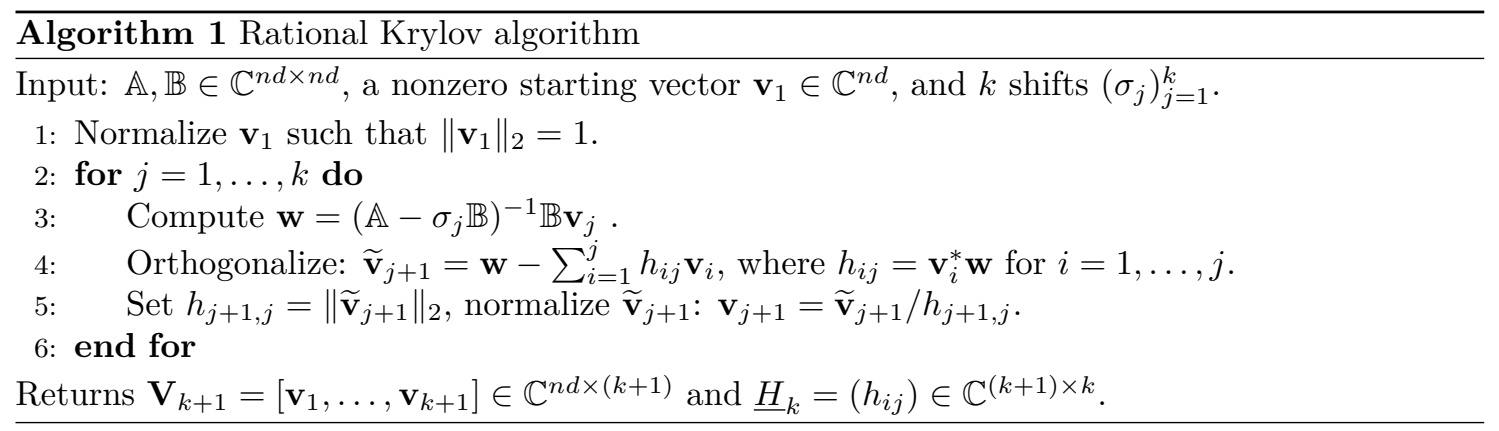

The matrix $\mathbf{V}_{k+1}$ satisfies the rational Krylov recurrence relation

$$
\mathbb{A} \mathbf{V}_{k+1} \underline{H}_{k}=\mathbb{B} \mathbf{V}_{k+1} \underline{K}_{k},
$$

where $\underline{H}_{k}, \underline{K}_{k} \in \mathbb{C}^{(k+1) \times k}$ are upper Hessenberg matrices with $\underline{H}_{k}=\left(h_{i j}\right)$ and $\underline{K}_{k}=\underline{I}_{k}+$ $\underline{H}_{k} \operatorname{diag}\left(\sigma_{1}, \ldots, \sigma_{k}\right)$. The Ritz pairs $\left(\lambda_{i}, \mathbf{x}_{i}\right)$ of $\mathbb{A}-s \mathbb{B}$ can then be obtained from the eigenpairs 
$\left(\lambda_{i}, s_{i}\right)$ of the generalized eigenvalue problem

$$
K_{k} s_{i}=\lambda_{i} H_{k} s_{i}, \quad s_{i} \neq 0,
$$

where $H_{k}, K_{k}$ are the $k \times k$ upper parts of $\underline{H}_{k}$ and $\underline{K}_{k}$ by setting $\mathbf{x}_{i}=\mathbf{V}_{k+1} \underline{H}_{k} s_{i}$.

An orthonormal basis $\mathbf{Z}_{k+1}=\left[\mathbf{z}_{1}, \ldots, \mathbf{z}_{k+1}\right]$ for the left Krylov space associated with the pencil $\mathbb{A}-s \mathbb{B}$ is obtained by applying Algorithm ?? to $\mathbb{A}^{T}$ and $\mathbb{B}^{T}$. A two-sided rational Krylov method is obtained by applying Algorithm ?? to both $\mathbb{A}-s \mathbb{B}$ and $\mathbb{A}^{T}-s \mathbb{B}^{T}$. We then use the computed orthonormal bases $\mathbf{V}_{k+1}$ and $\mathbf{Z}_{k+1}$ to project $\mathbb{A}-s \mathbb{B}$ onto a smaller pencil

$$
A-s B=\mathbf{Z}_{k+1}^{T}(\mathbb{A}-s \mathbb{B}) \mathbf{V}_{k+1}
$$

of size $(k+1) \times(k+1)$. The right and left Ritz vectors $\mathbf{x}$ and $\mathbf{y}$ of $\mathbb{A}-s \mathbb{B}$ are spanned by $\mathbf{V}_{k+1}$ and $\mathbf{Z}_{k+1}$, respectively, i.e., $\mathbf{x}=\mathbf{V} \hat{x}$ and $\mathbf{y}=\overline{\mathbf{Z}} \hat{y}$, where $\hat{x}$ and $\hat{y}$ are right and left eigenvectors of $A-s B$, respectively. The triplet $(\lambda, \mathbf{x}, \mathbf{y})$ forms a desired approximate eigenvalue-eigenvector triplet for $\mathbb{A}-s \mathbb{B}$.

The size $n d$ of the iteration vectors in Algorithm ?? is large, which can lead to a heavy memory burden and an expensive orthogonalization process. Also, solving the large scale system in Step 3 of Algorithm ??, without exploiting its structure, is prohibitively expensive in memory for large $n$. To alleviate these, we use the Kronecker structure of the pencil $\mathbb{A}-s \mathbb{B}$ to express the iteration vectors in a compact format that considerably reduces the memory cost, and the cost of the GramSchmidt orthogonalization process. We describe this compact format in Section ?? and explain how to compute the iteration vectors efficiently, which includes the efficient solution of the linear system in Step 3 of Algorithm ??. Section ?? explains how to make use of the compact form of the vectors to compute the inner products needed for the Gram-Schmidt orthogonalization process. We present the compact rational Krylov algorithm in Section ??. Section ?? explains how to efficiently compute the reduced eigenvalue problem (??). Section ?? presents further reductions on the memory consumption and computational cost. We close the section with a comment on implicit filtering in Section ??, which we need for improving the numerical stability of the two-sided method.

3.1. Compact form of the iteration vectors. We show that the iteration vectors $\mathbf{w}$ and $\mathbf{v}_{j}$ in Algorithm ?? can be written in a compact form involving Kronecker products. Consider the pencil $\mathbb{A}-s \mathbb{B}$ in (??), associated with the expression for $P(s)$ in (??), and the solution w to the linear system

$$
(\mathbb{A}-s \mathbb{B}) \mathbf{w}=\mathbb{B} \mathbf{v},
$$

for some shift $s$, such that $\mathbb{A}-s \mathbb{B}$ is nonsingular. Consider also vectors $\mathbf{v}$ and $\mathbf{w}$ of length $n d$ that we partition as follows:

$$
\mathbf{v}=\left[\begin{array}{c}
\mathbf{v}^{[\phi]} \\
\mathbf{v}^{[\psi]}
\end{array}\right] \quad, \quad \mathbf{w}=\left[\begin{array}{c}
\mathbf{w}^{[\phi]} \\
\mathbf{w}^{[\psi]}
\end{array}\right]
$$

with $\mathbf{v}^{[\phi]}, \mathbf{w}^{[\phi]} \in \mathbb{C}^{n\left(d_{\phi}+1\right)}$ and $\mathbf{v}^{[\psi]}, \mathbf{w}^{[\psi]} \in \mathbb{C}^{n d_{\psi}}$. The vector $\mathbf{v}^{[\phi]}$ is itself partitioned into $d_{\phi}+1$ vectors $v^{[0]}, \ldots, v^{\left[d_{\phi}\right]}$ of length $n$, and $\mathbf{v}^{[\psi]}$ consists of the $d_{\psi}$ vectors $v^{\left[d_{\phi}+1\right]}, \ldots, v^{\left[d_{\phi}+d_{\psi}\right]}$ of length $n$.

Assume that the conditions of Lemma ?? with respect to $M_{d_{\psi}}-s N_{d_{\psi}}, M_{d_{\phi}}-s N_{d_{\phi}}$ and $\phi_{0}(s)$ are satisfied. By Theorem ??, if $s$ is not an eigenvalue of $\mathbb{A}-s \mathbb{B}$, then it is not an eigenvalue of $P(s)$ so that $P(s)$ is nonsingular. We can transform the linear system (??) to an equivalent system by multiplying it on the left with

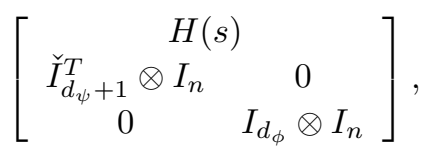


where $H(s)$ is as in Lemma ??(ii) and is assumed to be of full row rank. By using Lemma ??(ii) to replace $H(s)(\mathbb{A}-s \mathbb{B})$ with $e_{1}^{T} \otimes g(s) P(s)$, we obtain the linear system

$$
\begin{aligned}
& {\left[\begin{array}{cc}
e_{1}^{T} \otimes g(s) P(s) & 0 \\
\left(\check{I}_{d_{\psi}+1}^{T} \otimes I_{n}\right)(\mathbf{A}-s \mathbf{B}) & \check{I}_{d_{\psi}+1}^{T}\left(M_{d_{\psi}}-s N_{d_{\psi}}\right)^{T} \otimes I_{n} \\
\left(M_{d_{\phi}}-s N_{d_{\phi}}\right) \otimes I_{n} & 0
\end{array}\right]\left[\begin{array}{l}
\mathbf{w} \\
\mathbf{w}[\phi]
\end{array}\right]=} \\
& {\left[\begin{array}{cc}
{[\psi]} \\
\check{I}_{d_{\psi}+1}^{T} \otimes I_{n} & 0 \\
0 & I_{d_{\phi}} \otimes I_{n}
\end{array}\right]\left[\begin{array}{cc}
\mathbf{B} & N_{d_{\psi}}^{T} \otimes I_{n} \\
N_{d_{\phi}} \otimes I_{n} & 0
\end{array}\right]\left[\begin{array}{c}
\mathbf{v}[\phi] \\
\mathbf{v}[\psi]
\end{array}\right] . }
\end{aligned}
$$

The solution $\mathbf{w}$ to (??) can be obtained as follows:

$$
\begin{aligned}
w^{[0]}= & g(s)^{-1} P(s)^{-1} H(s) \mathbb{B} \mathbf{v}, \\
\left(\check{I}_{d_{\phi}+1}^{T} \otimes I_{n}\right) \mathbf{w}^{[\phi]}= & \left(E_{\phi}(s) N_{d_{\phi}} \otimes I_{n}\right) \mathbf{v}^{[\phi]}-E_{\phi}(s)\left(M_{d_{\phi}}-s N_{d_{\phi}}\right) e_{1} \otimes w^{[0]}, \\
\mathbf{w}^{[\psi]}= & \left(F_{\psi}(s)^{T} \otimes I_{n}\right) \mathbf{B} \mathbf{v}^{[\phi]}+\left(\left(N_{d_{\psi}} F_{\psi}(s)\right)^{T} \otimes I_{n}\right) \mathbf{v}^{[\psi]} \\
& \quad-\left(F_{\psi}(s)^{T} \otimes I_{n}\right)(\mathbf{A}-s \mathbf{B}) \mathbf{w}^{[\phi]},
\end{aligned}
$$

where

$$
E_{\phi}(s)=\left(\left(M_{d_{\phi}}-s N_{d_{\phi}}\right) \check{I}_{d_{\phi}+1}\right)^{-1}, \quad F_{\psi}(s)=\check{I}_{d_{\psi}+1}\left(\left(M_{d_{\psi}}-s N_{d_{\psi}}\right) \check{I}_{d_{\psi}+1}\right)^{-1} .
$$

In what follows, we will repeatedly use the fact that if $\mathbf{a}=\left(X \otimes I_{n}\right) \mathbf{b}$ for some $\mathbf{a}, \mathbf{b} \in \mathbb{C}^{d n}$ and $X \in \mathbb{C}^{d \times d}$, then the $j$ th block $a^{[j]}$ of length $n$ of $\mathbf{a}$ is a linear combination of the $d$ blocks $b^{[1]}, b^{[2]}, \ldots, b^{[d]}$ of $\mathbf{b}$. We will need the following result.

Lemma 3.1. Let $r_{k}=\operatorname{rank} \widetilde{V}_{k}$, where

$$
\widetilde{V}_{k}=\left[v_{1}^{[0]}, \ldots, v_{1}^{\left[d_{\phi}\right]}, v_{2}^{[0]}, \ldots, v_{2}^{\left[d_{\phi}\right]}, \ldots, v_{k}^{[0]}, \ldots, v_{k}^{\left[d_{\phi}\right]}\right] \in \mathbb{C}^{n \times\left(d_{\phi}+1\right) k},
$$

with $v_{j}^{[\ell]}$ denoting the $(\ell+1)$ st block of length $n$ of the $j$ th column of the matrix $\mathbf{V}_{k}$ in Algorithm ??. Then,

(i) $r_{1} \leq \min \left(d_{\phi}+1, n\right)$,

(ii) $r_{k}=r_{k-1}$ or $r_{k}=r_{k-1}+1$ for $k>1$.

Proof. Statement (i) is obvious. Let us prove (ii). Let the columns of $Q_{k} \in \mathbb{C}^{n \times r_{k}}$ form an orthonormal basis for the column range of $\widetilde{V}_{k}$. Since $\mathbf{v}_{k}$ for $k>1$ is a linear combination of $\mathbf{w}_{k-1}$ and $\mathbf{v}_{1}, \ldots, \mathbf{v}_{k-1}$ (see step 4 of Algorithm ??), the blocks $v_{k}^{[i]}, i=0, \ldots, d_{\phi}$, of $\mathbf{v}_{k}^{[\phi]}$ lie in the column range of $\left[Q_{k-1}, w_{k-1}^{[0]}, \ldots, w_{k-1}^{\left[d_{\phi}\right]}\right]$. But, by (??), with $\mathbf{w}^{[\phi]} \equiv \mathbf{w}_{k-1}^{[\phi]}$ and $\mathbf{v}^{[\phi]} \equiv \mathbf{v}_{k-1}^{[\phi]}$, we see that $w_{k-1}^{[j]}, 1 \leq j \leq d_{\phi}$ is a linear combination of $v_{k-1}^{[0]}, v_{k-1}^{[1]}, \ldots, v_{k-1}^{\left[d_{\phi}\right]}$, and $w_{k-1}^{[0]}$, so that

$$
\operatorname{range}\left(Q_{k}\right)=\operatorname{range}\left(\left[Q_{k-1}, w_{k-1}^{[0]}, \ldots, w_{k-1}^{\left[d_{\phi}\right]}\right]\right)=\operatorname{range}\left(\left[Q_{k-1}, w_{k-1}^{[0]}\right]\right),
$$

i.e., $r_{k}$ can be at most $r_{k-1}+1$, which proves the lemma.

We introduce the auxiliary matrix

$$
L_{k}=\left[A_{0}, A_{1}, \ldots, A_{d_{\mathrm{AB}}-1}, B_{0}, B_{1}, \ldots, B_{d_{\mathrm{AB}}-1}\right]\left(I_{2 d_{\mathrm{AB}}} \otimes Q_{k}\right) \in \mathbb{C}^{n \times 2 d_{\mathrm{AB}} r_{k}},
$$

where $Q_{k} \in \mathbb{C}^{n \times r_{k}}$ is the matrix with orthonormal columns defined in the proof of Lemma ?? and $d_{\mathrm{AB}}$ is the number of nonzero $n \times n$ blocks in the pencil $\mathbf{A}-s \mathbf{B}$, used to define $\mathbb{A}-s \mathbb{B}$.

THEOREM 3.2 (Compact form of iteration vector $\mathbf{v}_{k}$ ). Let $\mathbf{v}_{k} \in \mathbb{C}^{n d}, k>1$ be the iteration vector computed at step $k-1$ of the rational Krylov algorithm applied to the pair $(\mathbb{A}, \mathbb{B})$ associated with $P(s)$ in (??). Assume that $s=\sigma_{k}$ is such that $\mathbb{A}-s \mathbb{B}$ is nonsingular and the conditions of Lemma ?? with respect to $M_{d_{\psi}}-s N_{d_{\psi}}, M_{d_{\phi}}-s N_{d_{\phi}}$ and $\phi_{0}(s)$ are satisfied. Then,

$$
\mathbf{v}_{k}^{[\phi]}=\left(I_{d_{\phi}+1} \otimes Q_{k}\right) \mathbf{u}_{k}^{[\phi]} \text { for some } \mathbf{u}_{k}^{[\phi]} \in \mathbb{C}^{r_{k}\left(d_{\phi}+1\right)},
$$


where $r_{k}=\operatorname{rank} \widetilde{V}_{k}$ with $\widetilde{V}_{k}$ as in (??) and the columns of $Q_{k} \in \mathbb{C}^{n \times r_{k}}$ form an orthonormal basis for range $\left(\widetilde{V}_{k}\right)$. Moreover, with $L_{k}$ as in (??), if $d_{\psi} \neq 0$ and the lower part of the initial vector $\mathbf{v}_{1}^{[\psi]}$ is chosen to be of the form $\mathbf{v}_{1}^{[\psi]}=\left(I_{d_{\psi}} \otimes L_{1}\right) \mathbf{u}_{1}^{[\psi]}$ for some $\mathbf{u}_{1}^{[\psi]} \in \mathbb{C}^{2 r_{1} d_{A B} d_{\psi}}$ then

$$
\mathbf{v}_{k}^{[\psi]}=\left(I_{d_{\psi}} \otimes L_{k}\right) \mathbf{u}_{k}^{[\psi]} \text { for some } \mathbf{u}_{k}^{[\psi]} \in \mathbb{C}^{2 r_{k} d_{A B} d_{\psi}} .
$$

Proof. The compact form for $\mathbf{v}_{k}^{[\phi]}$ follows from Lemma ?? and its proof. For $\mathbf{v}_{k}^{[\psi]}$, we proceed by induction on $k$. The compact form clearly holds for $k=1$. Assume it holds for $k-1$, i.e., $\mathbf{v}_{k-1}^{[\psi]}=\left(I_{d_{\psi}} \otimes L_{k-1}\right) \mathbf{u}_{k-1}^{[\psi]}$ for some $\mathbf{u}_{k-1}^{[\psi]} \in \mathbb{C}^{2 r_{k-1} d_{A B} d_{\psi}}$. From Algorithm ??, $\mathbf{v}_{k}^{[\psi]}=\alpha \mathbf{w}_{k-1}^{[\psi]}-$ $\alpha \sum_{i=1}^{k-1} h_{i j} \mathbf{v}_{i}^{[\psi]}$ with $\alpha$ such that $\left\|\mathbf{v}_{k}\right\|_{2}=1$. Now, from (??)-(??) and the induction hypothesis, for the two first terms in the expression for $\mathbf{w}_{k-1}^{[\psi]}$ in (??), it is easy to see that

$$
\left(F_{\psi}(s)^{T} \otimes I_{n}\right) \mathbf{B v}_{k-1}^{[\phi]}+\left(\left(N_{d_{\psi}} F_{\psi}(s)\right)^{T} \otimes I_{n}\right) \mathbf{v}_{k-1}^{[\psi]}=\left(I_{d_{\psi}} \otimes L_{k-1}\right) \mathbf{a}
$$

for some $\mathbf{a} \in \mathbb{C}^{2 r_{k-1} d_{A B} d_{\psi}}$. It follows from (??)-(??) and (??) that

$$
\mathbf{w}_{k-1}^{[\phi]}=\left(I_{d_{\phi}+1} \otimes Q_{k}\right) \mathbf{t}_{k}^{[\phi]} \text { for some } \mathbf{t}_{k}^{[\phi]} \in \mathbb{C}^{r_{k}\left(d_{\phi}+1\right)}
$$

so that $\left(F_{\psi}(s)^{T} \otimes I_{n}\right)(\mathbf{A}-s \mathbf{B}) \mathbf{w}_{k-1}^{[\phi]}=\left(I_{d_{\psi}} \otimes L_{k}\right) \mathbf{b}$ for some $\mathbf{b} \in \mathbb{C}^{2 r_{k} d_{A B} d_{\psi}}$. Hence,

$$
\mathbf{w}_{k}^{[\psi]}=\left(I_{d_{\psi}} \otimes L_{k}\right) \mathbf{t}_{k}^{[\psi]} \text { for some } \mathbf{t}_{k}^{[\psi]} \in \mathbb{C}^{2 r_{k} d_{A B} d_{\psi}}
$$

and $\mathbf{v}_{k}^{[\psi]}=\alpha \mathbf{w}_{k-1}^{[\psi]}-\alpha \sum_{i=1}^{k-1} h_{i j} \mathbf{v}_{i}^{[\psi]}=\left(I_{d_{\psi}} \otimes L_{k}\right) \mathbf{u}_{k}^{[\psi]}$ for some $\mathbf{u}^{[\psi]} \in \mathbb{C}^{2 r_{k} d_{A B} d_{\psi}}$, which concludes the proof.

For the compact forms (??)-(??) to hold, $\mathbf{v}_{1}^{[\psi]}$ must lie in the column range of $L_{1}$. Recall that, by Lemma ??, the size of $Q_{k}$ increases by at most one at each iteration and that of $L_{k}$ by at most $2 d_{A B}$ so that $L_{k}$ can end up having a large number of columns. We explain in Section ?? how to reduce the number of columns of $L_{k}$, which can be as little as $m r_{k}$, where $m$ is the number of matrices defining the nonlinear eigenvalue problem via the matrix-valued function in (??) and is usually small. Now, with an appropriate choice of the starting vector $\mathbf{v}_{1}, r_{1}=\operatorname{rank}\left(Q_{1}\right)=1$ and $Q_{k}$ has at most rank $r_{k}=k$. So we encourage the reader to think of $Q_{k}$ as being $n \times k$ and $L_{k}$ to be $n$ times a small multiple of $k$.

We drop the subscript $k$ in what follows and write

$$
\mathbf{v}=\left[\begin{array}{c}
\mathbf{v}^{[\phi]} \\
\mathbf{v}^{[\psi]}
\end{array}\right]=\left[\begin{array}{c}
\left.\left(I_{d_{\phi}+1} \otimes Q\right) \mathbf{u}^{[\phi]}\right] \\
\left(I_{d_{\psi}} \otimes L\right) \mathbf{u}^{[\psi]}
\end{array}\right], \quad \mathbf{w}=\left[\begin{array}{c}
\mathbf{w}^{[\phi]} \\
\mathbf{w}^{[\psi]}
\end{array}\right]=\left[\begin{array}{c}
\left(I_{d_{\phi}+1} \otimes Q\right) \mathbf{t}^{[\phi]} \\
\left(I_{d_{\psi}} \otimes L\right) \mathbf{t}^{[\psi]}
\end{array}\right]
$$

for some $\mathbf{u}^{[\phi]}, \mathbf{t}^{[\phi]} \in \mathbb{C}^{r\left(d_{\phi}+1\right)}$ and some $\mathbf{u}^{[\psi]}, \mathbf{t}^{[\psi]} \in \mathbb{C}^{2 r d_{A B} d_{\psi}}$, and where $r$ is the rank of $Q$. We still have to present a practical way to compute the short vectors $\mathbf{t}^{[\phi]}$ and $\mathbf{t}^{[\psi]}$, and then $\mathbf{u}^{[\phi]}, \mathbf{u}^{[\psi]}$ defining the next iteration vector $\mathbf{v}$. Note that, since $L$ is not a full rank matrix, $\mathbf{u}^{[\psi]}$ and $\mathbf{t}^{[\psi]}$ are not unique.

To compute $\mathbf{t}$, it is convenient to introduce $\mathbf{A}_{L}, \mathbf{B}_{L} \in \mathbb{C}^{2 r d_{\mathrm{AB}}\left(d_{\psi}+1\right) \times r\left(d_{\phi}+1\right)}$ such that

$$
\mathbf{A}\left(I_{d_{\phi}+1} \otimes Q\right)=\left(I_{d_{\psi}+1} \otimes L\right) \mathbf{A}_{L} \quad \mathbf{B}\left(I_{d_{\phi}+1} \otimes Q\right)=\left(I_{d_{\psi}+1} \otimes L\right) \mathbf{B}_{L} .
$$

The matrices $\mathbf{A}_{L}$ and $\mathbf{B}_{L}$ are in general of much smaller dimension than $\mathbf{A}$ and $\mathbf{B}$. Note that for $\mathbf{A}, \mathbf{B}$ in (??) for which $d_{A B}=d=d_{\phi}+1$, we have that

$$
\mathbf{A}_{L}=\left[e_{1}, e_{2}, \ldots, e_{d_{A B}}\right] \otimes I_{r}, \quad \mathbf{B}_{L}=\left[e_{d_{A B}+1}, e_{d_{A B}+2}, \ldots, e_{2 d_{A B}}\right] \otimes I_{r},
$$

and for $\mathbf{A}, \mathbf{B}$ in (??) for which $d_{A B}=d_{\phi}+1=d_{\psi}+1$, we have

$$
\mathbf{A}_{L}=\left[\begin{array}{cccc}
e_{1} & & & \\
& e_{2} & & \\
& & \ddots & \\
& & & e_{d_{\psi}+1}
\end{array}\right] \otimes I_{r}, \quad \mathbf{B}_{L}=\left[\begin{array}{llll}
e_{d_{\psi}+2} & & & \\
& e_{d_{\psi}+3} & & \\
& & \ddots & \\
& & & e_{2 d_{\psi}+2}
\end{array}\right] \otimes I_{r},
$$


where $e_{j}$ is the $j$ th column of the $2 d_{A B} \times 2 d_{A B}$ identity matrix.

If $\mathbf{w}$ is the solution to the linear system $(\mathbb{A}-s \mathbb{B}) \mathbf{w}=\mathbb{B} \mathbf{v}$ at iteration $k$ of Algorithm ?? then the $Q$ in the compact form for $\mathbf{w}$ in (??) corresponds to $Q_{k+1}$, which has not been formed at this point. It is then necessary to compute $w^{[0]}$ as the solution to the linear system in (??) first and update $Q$ accordingly (recall that range $\left.\left(Q_{k+1}\right)=\operatorname{range}\left(\left[Q_{k}, w^{[0]}\right]\right)\right)$. But the $Q$ in the compact form for $\mathbf{v}$ is $Q_{k}$, which is known at this stage. So we can take advantage of the compact form of $\mathbf{v}$ to reduce the cost of the computation of $w^{[0]}$, starting with the right-hand side $H(s) \mathbb{B} \mathbf{v}$, which we rewrite as

$$
b:=H(s) \mathbb{B} \mathbf{v}=L H_{L}(s) \mathbb{B}_{L} \mathbf{u},
$$

where

$$
H_{L}(s)=\phi_{0}(s)\left(\Psi_{d_{\psi}}(s)^{T} \otimes I_{2 r d_{A B}}\right)\left[I_{2 r d_{A B}\left(d_{\psi}+1\right)} \quad\left(\mathbf{A}_{L}-s \mathbf{B}_{L}\right)\left(F_{\phi}(s) \otimes I_{r}\right)\right]
$$

with $F_{\phi}(s)$ as in (??) (and $\phi$ in place of $\psi$ ), and

$$
\mathbb{B}_{L}=\left[\begin{array}{cc}
\mathbf{B}_{L} & N_{d_{\psi}}^{T} \otimes I_{2 r d_{A B}} \\
N_{d_{\phi}} \otimes I_{r} & 0
\end{array}\right] .
$$

This follows from (??) and the fact that

$$
\left(N_{d_{\phi}} \otimes I_{n}\right)\left(I_{d_{\phi}+1} \otimes Q\right)=\left(I_{d_{\phi}} \otimes Q\right)\left(N_{d_{\phi}} \otimes I_{r}\right), \quad\left(N_{d_{\psi}}^{T} \otimes I_{n}\right)\left(I_{d_{\psi}} \otimes L\right)=\left(I_{d_{\psi}+1} \otimes L\right)\left(N_{d_{\psi}}^{T} \otimes I_{2 r d_{A B}}\right) .
$$

Now, replacing $\mathbf{v}^{[\phi]}$ and $\mathbf{v}^{[\psi]}$ in (??)-(??) by their compact form in (??), using (??) and $Q^{*} Q=I_{r}$, a straightforward computation shows that

$$
\begin{aligned}
t^{[0]} & =Q^{*} w^{[0]} \\
\left(\check{I}_{d_{\phi}+1}^{T} \otimes I_{r}\right) \mathbf{t}^{[\phi]} & =\left(E_{\phi}(s) N_{d_{\phi}} \otimes I_{r}\right) \mathbf{u}^{[\phi]}-E_{\phi}(s)\left(M_{d_{\phi}}-s N_{d_{\phi}}\right) e_{1} \otimes t^{[0]} \\
\mathbf{t}^{[\psi]} & =\left(F_{\psi}(s)^{T} \otimes I_{2 r d_{A B}}\right)\left(\mathbf{B}_{L}\left(\mathbf{u}^{[\phi]}+s \mathbf{t}^{[\phi]}\right)-\mathbf{A}_{L} \mathbf{t}^{[\phi]}\right)+\left(N_{d_{\psi}} F_{\psi}(s)^{T} \otimes I_{2 r d_{A B}}\right) \mathbf{u}^{[\psi]} .
\end{aligned}
$$

As mentioned before, the expression for $\mathbf{t}^{[\psi]}$ is not unique but $\mathbf{t}^{[\psi]}$ defines $\mathbf{w}^{[\psi]}$ uniquely.

In what follows we provide simpler expressions for $H_{L}(s)$ in (??) and $\mathbf{t}$ in (??) that are also easier to compute. We introduce the matrix $\mathbb{A}_{L}$ that has the structure of $\mathbb{B}_{L}$ in (??) but with $\mathbf{A}_{L}, M_{d_{\psi}}^{T}$ and $M_{d_{\phi}}$ in place of $\mathbf{B}_{L}, N_{d_{\psi}}^{T}$ and $N_{d_{\phi}}$, respectively. We will also need

$$
\check{\mathbb{I}}_{L}=\left[\begin{array}{ll}
\check{I}_{d_{\psi}+1}^{T} \otimes I_{2 r d_{\mathrm{AB}}} & \\
& I_{d_{\phi}} \otimes I_{r}
\end{array}\right] \quad \text { and } \quad \check{\mathbb{I}}_{R}=\left[\begin{array}{ll}
\check{I}_{d_{\phi}+1} \otimes I_{r} & \\
& I_{d_{\psi}} \otimes I_{2 r d_{\mathrm{AB}}}
\end{array}\right] .
$$

Using an approach similar to the one we used in the proof of Lemma ??, we can show that if we let

$$
P_{L}(s)=\left(\Psi_{d_{\psi}}(s) \otimes I_{2 r d_{\mathrm{AB}}}\right)^{T}\left(\mathbf{A}_{L}-s \mathbf{B}_{L}\right)\left(\Phi_{d_{\phi}}(s) \otimes I_{r}\right)
$$

then the $r\left(2 d_{\mathrm{AB}}\left(d_{\psi}+1\right)+d_{\phi}\right) \times r\left(2 d_{\mathrm{AB}} d_{\psi}+\left(d_{\phi}+1\right)\right)$ pencil $\mathbb{A}_{L}-s \mathbb{B}_{L}$ and the $2 r d_{\mathrm{AB}} \times r$ rational matrix function $P_{L}(s)$ are related by the one-sided factorization

$$
H_{L}(s)\left(\mathbb{A}_{L}-s \mathbb{B}_{L}\right)=e_{1}^{T} \otimes P_{L}
$$

with $H_{L}(s)$ as in (??). Now if we combine the first $2 r d_{\mathrm{AB}}$ columns of equation (??) to equation (??) with its first $r$ columns removed we obtain that $H_{L}(s)$ is a solution to the linear system

$$
H_{L}(s)\left[\left[\begin{array}{c}
I_{2 r d_{\mathrm{AB}}} \\
0
\end{array}\right] \quad\left(\mathbb{A}_{L}-s \mathbb{B}_{L}\right) \check{\mathbb{I}}_{R}\right]=\phi_{0} \psi_{0}\left[\begin{array}{ll}
I_{2 r d_{\mathrm{AB}}} & 0
\end{array}\right] .
$$

The matrix on the right-hand side of $H_{L}(s)$ is block upper triangular with nonsingular diagonal blocks. This follows from the fact that $\check{I}_{d_{\psi}+1}^{T}\left(M_{d_{\psi}}-s N_{d_{\psi}}\right)^{T}$ and $\left(M_{d_{\phi}}-s N_{d_{\phi}}\right) \check{I}_{d_{\phi}+1}$ have full 
rank. Hence $H_{L}(s)$ is uniquely determined by (??). It is not difficult to check that $\mathbf{t}$ in (??) is a solution to the $\left(2 r d_{\mathrm{AB}} d_{\psi}+r\left(d_{\phi}+1\right)\right) \times\left(2 r d_{\mathrm{AB}} d_{\psi}+r\left(d_{\phi}+1\right)\right)$ block lower triangular linear system

$$
\left[\begin{array}{c}
e_{1}^{T} \otimes I_{r} \\
\check{\mathbb{I}}_{L}\left(\mathbb{A}_{L}-s \mathbb{B}_{L}\right)
\end{array}\right]\left[\begin{array}{c}
\mathbf{t}^{[\phi]} \\
\mathbf{t}^{[\psi]}
\end{array}\right]=\left[\begin{array}{c}
Q^{*} w^{[0]} \\
\check{\mathbb{I}}_{L} \mathbb{B}_{L} \mathbf{u}
\end{array}\right]
$$

That the matrix of this linear system is nonsingular follows from the same arguments we used to prove nonsingularity of the system (??). Hence $\mathbf{t}$ is uniquely determined by (??). This presents an alternative to the explicit expression for $\mathbf{t}$ in (??). Note that solving (??), using a sparse direct linear solver, e.g., is, in general, not cheaper than (??), but it is less challenging than working out (??).

3.2. Orthogonalization. We now describe how to implement the inner product of $\mathbf{v}$ and $\mathbf{w}$ in (??) needed in the Gram-Schmidt orthogonalization in Step 4 of Algorithm ??. Since $Q$ has orthonormal columns,

$$
\mathbf{v}^{*} \mathbf{w}=\left(\mathbf{u}^{[\phi]}\right)^{*} \mathbf{t}^{[\phi]}+\left(\left[A_{0} Q, \ldots, B_{d_{\mathrm{AB}}-1} Q\right] \mathbf{u}^{[\psi]}\right)^{*}\left(\left[A_{0} Q, \ldots, B_{d_{\mathrm{AB}}-1} Q\right] \mathbf{t}^{[\psi]}\right)
$$

where the second term in the summation disappears when $d_{\psi}=0$ (e.g., for the CORK pencil $\mathbf{L}_{1}$ in (??)) but has a non negligible cost to compute when $d_{\psi} \neq 0$. In the latter case we propose to replace the Euclidean inner product $\mathbf{v}^{*} \mathbf{w}$ with a quasi inner product that is cheaper to evaluate. We consider both

$$
\langle\mathbf{v}, \mathbf{w}\rangle_{\phi}=\left(\mathbf{u}^{[\phi]}\right)^{*} \mathbf{t}^{[\phi]} \text { and }\langle\mathbf{v}, \mathbf{w}\rangle_{\phi, \psi}=\left[\begin{array}{l}
\mathbf{u}^{[\phi]} \\
\mathbf{u}^{[\psi]}
\end{array}\right]^{*}\left[\begin{array}{l}
\mathbf{t}^{[\phi]} \\
\mathbf{t}^{[\psi]}
\end{array}\right]
$$

These are both quasi inner products since $\langle\mathbf{v}, \mathbf{v}\rangle_{\phi}=0$ does not imply $\mathbf{v}=0$ and when $L$ is not of full column rank, there exists $\mathbf{u}^{[\psi]} \neq 0$ such that $\mathbf{v}=0$ but $\langle\mathbf{v}, \mathbf{v}\rangle_{\phi, \psi} \neq 0$. The latter situation should not lead to unlucky breakdown. We compare numerically the two quasi inner products in (??) in Section ??.

3.3. The overall algorithm. The compact rational Krylov algorithm is presented in Algorithm ??. Similarly to Algorithm ??, Algorithm ?? applied to the transposes of $\mathbb{A}$ and $\mathbb{B}$ returns a basis $\mathbf{Z}$ in compact format for the left Krylov subspace. Note that, with regards to numerical stability, we use reorthogonalization for both the orthogonalization with Euclidean inner product in Step 6 and for the orthogonalization with the alternative inner products in Steps 13 and 14 [?].

Algorithm ?? requires the storage of $Q$, and $\mathbf{u}_{1}, \ldots, \mathbf{u}_{k+1}$. Recall that $L$ is not stored. Assume that the starting vector takes the form (??), defined in Section ??, which implies $r_{1}=1$. Storing $k$ iteration vectors in the compact format requires $O\left(n k+k^{2}\left(d_{\phi}+1+2 d_{\mathrm{AB}} d_{\psi}\right)\right)$ floating point numbers, compared to $O\left(n k\left(d_{\phi}+d_{\psi}+1\right)\right)$ for the full unstructured vector format. Note that the compact format is only beneficial for sufficiently large scale problems. The orthogonalization cost when the $\langle\cdot, \cdot\rangle_{\phi}$ inner product is used, is $O\left(n k^{2}\right)+O\left(\left(d_{\phi}+1\right) k^{2}\right)$ floating point operations as for one-sided CORK. For the $\langle\cdot, \cdot\rangle_{\phi, \psi}$ inner product, the cost is $O\left(n k^{2}\right)+O\left(\left(d_{\phi}+1+2 d_{\mathrm{AB}} d_{\psi}\right) k^{2}\right)$ floating point operations.

3.4. Computation of the projection. Let $\mathbf{V}$ be the basis in compact form returned by Algorithm ?? applied to $\mathbb{A}-s \mathbb{B}$ and let $\mathbf{Z}$ be the basis in compact form returned by Algorithm ?? when applied to $\mathbb{A}^{T}-s \mathbb{B}^{T}$,

$$
\mathbf{V}=\left[\begin{array}{ll}
I_{d_{\phi}+1} \otimes Q_{V} & \\
& I_{d_{\psi}} \otimes L_{V}
\end{array}\right]\left[\begin{array}{c}
\mathbf{U}_{V}^{[\phi]} \\
\mathbf{U}_{V}^{[\psi]}
\end{array}\right], \quad \mathbf{Z}=\left[\begin{array}{ll}
I_{d_{\psi}+1} \otimes Q_{Z} & \\
& I_{d_{\phi}} \otimes L_{Z}
\end{array}\right]\left[\begin{array}{c}
\mathbf{U}_{Z}^{[\psi]} \\
\mathbf{U}_{Z}^{[\phi]}
\end{array}\right] .
$$




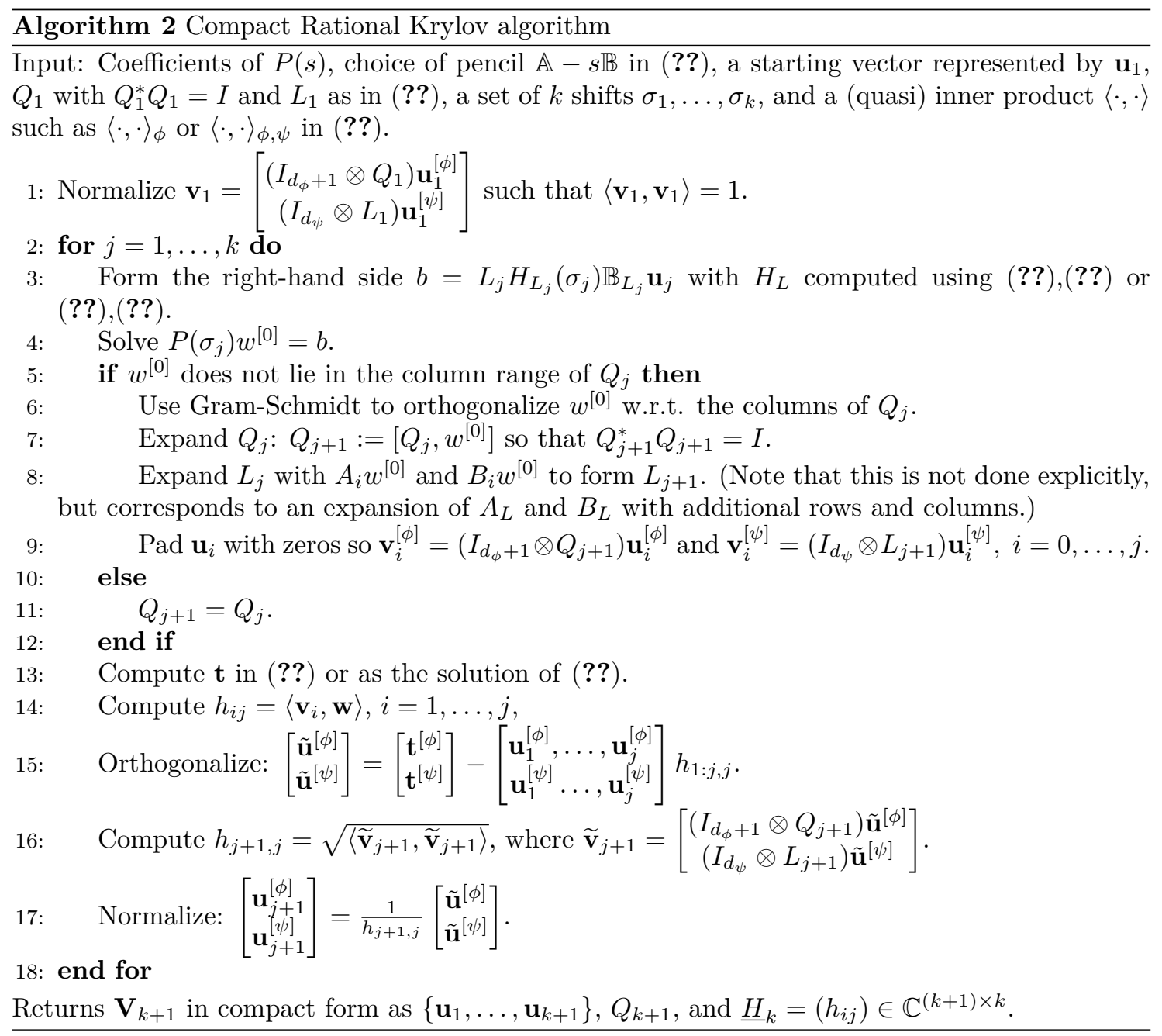

Here, note that the bases $\Psi$ and $\Phi$ are swapped for $\mathbf{Z}$, since $\mathbf{Z}$ is formed using $\mathbb{A}^{T}$ and $\mathbb{B}^{T}$. Then, we have

$$
\begin{aligned}
{\left[\begin{array}{ll}
I_{d_{\psi}+1} \otimes Q_{Z} & \\
& I_{d_{\phi}} \otimes L_{Z}
\end{array}\right]^{*}(\mathbb{A}-s \mathbb{B})\left[\begin{array}{ll}
I_{d_{\phi}+1} \otimes Q_{V} & \\
& I_{d_{\psi}} \otimes L_{V}
\end{array}\right] } \\
=\left[\begin{array}{ll}
I_{d_{\psi}+1} \otimes Q_{Z}^{*} L_{V} & \\
& I_{d_{\phi}} \otimes L_{Z}^{*} Q_{V}
\end{array}\right]\left(\mathbb{A}_{L}-\mathbb{B}_{L}\right)
\end{aligned}
$$

and, therefore

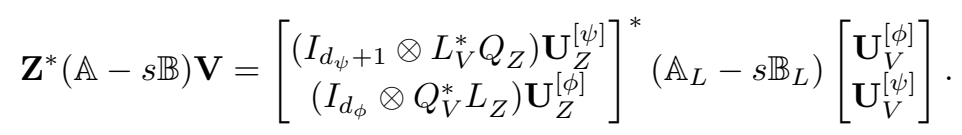

An efficient way to evaluate $Q_{Z}^{*} L_{V}$ and $L_{Z}^{*} Q_{V}$ is to first compute $\hat{A}_{i}=Q_{Z}^{*} A_{i} Q_{V}$ and $\hat{B}_{i}=$ $Q_{Z}^{*} B_{i} Q_{V}$.

3.5. Reduction of the size of $L$. The size of $L$ and, consequently, $\mathbf{u}^{[\psi]}$, can be large, in particular, when $d_{\mathrm{AB}}$ is large. We show via examples that the number of nonzero columns of $L$ is usually much smaller than $2 r d_{\mathrm{AB}}$ and explain how to reduce the size of $L$ and as a consequence, the length of $\mathbf{u}^{[\psi]}$. 
EXAMPLE 3. Consider the matrix polynomial in the monomial basis from Example ??. The relation between the coefficient matrices $P_{i}, i=0, \ldots, d$ and the CORK coefficient matrices $A_{i}, B_{i}$ $i=0, \ldots, d-1$ is given by

$$
\left[A_{0}, \ldots, A_{d-1}, B_{0}, \ldots, B_{d-1}\right]=\left[P_{0}, \ldots, P_{d}\right]\left(T \otimes I_{n}\right) \quad \text { with } \quad T=\left[\begin{array}{ll}
\hat{I}_{d+1} & -e_{d+1} e_{d}^{T}
\end{array}\right] \in \mathbb{R}^{(d+1) \times 2 d},
$$

where $\hat{I}_{d+1}$ is the $(d+1) \times(d+1)$ identity matrix with its last column removed. For $d=3$, e.g., we have

$$
T=\left[\begin{array}{cccccc}
1 & 0 & 0 & 0 & 0 & 0 \\
0 & 1 & 0 & 0 & 0 & 0 \\
0 & 0 & 1 & 0 & 0 & 0 \\
0 & 0 & 0 & 0 & 0 & -1
\end{array}\right] \in \mathbb{R}^{4 \times 6}
$$

For any polynomial of degree $d$, a minimal formulation consists of at most $d+1$ different matrices $A_{i}, B_{i}$, so that $T$ has at most $d+1$ rows. Then

$$
\begin{aligned}
L=\left[A_{0}, \ldots, A_{d-1}, B_{0}, \ldots, B_{d-1}\right]\left(I_{2 d} \otimes Q\right) & =\left[P_{0}, \ldots, P_{d}\right]\left(T \otimes I_{n}\right)\left(I_{2 d} \otimes Q\right) \\
& =\left[P_{0}, \ldots, P_{d}\right]\left(I_{d+1} \otimes Q\right)\left(T \otimes I_{r}\right)=L_{G}\left(T \otimes I_{r}\right),
\end{aligned}
$$

where $L_{G}=\left[P_{0}, \ldots, P_{d}\right]\left(I_{d+1} \otimes Q\right) \in \mathbb{C}^{n \times r(d+1)}$ with $d+1<2 d_{\mathrm{AB}}$. The iteration vectors then take the form

$$
\mathbf{w}=\left[\begin{array}{c}
\left(I_{d_{\phi}+1} \otimes Q\right) \mathbf{u}^{[\phi]} \\
\left(I_{d_{\psi}} \otimes L_{G}\right) \mathbf{u}^{[\psi]}
\end{array}\right]
$$

where $\mathbf{u}^{[\psi]} \in \mathbb{C}^{r(d+1) d_{\psi}}$, which can be much shorter in length than $\mathbf{u}^{[\psi]}$ in (??). When we use $L_{G}$ instead of $L$, we also need to replace $A_{L}$ and $B_{L}$ by $A_{L, G}$ and $B_{L, G}$ respectively, such that

$$
\mathbf{A}\left(I_{d_{\phi}+1} \otimes Q\right)=\left(I_{d_{\psi}+1} \otimes L_{G}\right) A_{L, G}, \quad \mathbf{B}\left(I_{d_{\psi}+1} \otimes Q\right)=\left(I_{d_{\psi}+1} \otimes L_{G}\right) B_{L, G},
$$

where

$$
A_{L, G}=\left(I_{d_{\psi}+1} \otimes T \otimes I_{r}\right) A_{L}, \quad B_{L, G}=\left(I_{d_{\psi}+1} \otimes T \otimes I_{r}\right) B_{L} .
$$

The situation improves even more when the matrix coefficients of $P(s)$ are linear combinations of less than $d+1$ matrices. This is the case when $g(s) P(s)$ is the result of the polynomial or rational approximation of a nonlinear problem of the form (??) with $m \ll d$. The following example illustrates this.

EXAMPle 4. Consider the matrix polynomial with $m=3$ written as:

$$
P(s)=C_{0}+\left(s+2 s^{2}+3 s^{3}\right) C_{1}+s^{4} C_{2} .
$$

The relation between the coefficient matrices $C_{i}$ of $P$ and the CORK coefficient matrices in (??) is described by $\left[A_{0}, A_{1}, A_{2}, A_{3}, B_{0}, B_{1}, B_{2}, B_{3}\right]=\left[C_{0}, C_{1}, C_{2}\right]\left(T \otimes I_{n}\right)$, where

$$
T=\left[\begin{array}{cccccccc}
1 & 0 & 0 & 0 & 0 & 0 & 0 & 0 \\
0 & 1 & 2 & 3 & 0 & 0 & 0 & 0 \\
0 & 0 & 0 & 0 & 0 & 0 & 0 & -1
\end{array}\right] \in \mathbb{R}^{3 \times 8} .
$$

In Example ??, the size of $L$ is reduced from $n \times 2 r d_{\mathrm{AB}}$ to $n \times r m$ and $\mathbf{u}^{[\psi]}$ is reduced from size $2 r d_{\mathrm{AB}} d_{\psi}$ to $r m d_{\psi}$. In a similar way, the gun problem described in (??) is defined by the four coefficient matrices $K, M, W_{1}$ and $W_{2}$. If approximated by a rational function of degree 21 , as in Section ??, the resulting coefficient matrices $A_{i}$ and $B_{i}$ are just linear combinations of $K, M, W_{1}$ and $W_{2}$. Using the technique described above, we can reduce the size of $\mathbf{u}^{[\psi]}$ from $42 r d_{\psi}$ to $4 r d_{\psi}$. 
3.6. Implicit filter and numerical stability. Instabilities in the Lanczos method can sometimes be cured by an implicit filter [?]. Implicit filtering is also used for one-sided Krylov methods for reducing the chance of spurious eigenvalues [?], [?]. We recall the idea for the onesided rational Krylov method and then explain how it is applied to the two-sided method. The idea of implicit filtering for the rational Krylov method is due to [?].

The following theorem shows the case for constant shifts $\sigma_{1}=\cdots=\sigma_{k}$. For the general, but, more complicated case of different shifts, we refer to [?].

TheOREm 3.3. Let $\mathbf{V}_{k+1}, \underline{H}_{k}, \underline{K}_{k}$ satisfy (??). Define the 'skinny' QR factorization $\underline{H}_{k}=$ $\underline{Y}_{k} R_{k}$ where $\underline{Y}_{k} \in \mathbb{C}^{(k+1) \times k}$ has orthonormal columns and $R_{k} \in \mathbb{C}^{k \times k}$ is upper triangular. Define

$$
\mathbf{V}_{k}^{(F)}=\mathbf{V}_{k+1} \underline{Y}_{k} \in \mathbb{R}^{n \times k}, \quad \underline{H}_{k-1}^{(F)}=R_{k}\left(\check{I}_{k+1}^{T} \underline{Y}_{k} \check{I}_{k}\right) \in R^{k \times(k-1)}, \quad \underline{K}_{k-1}^{(F)}=\underline{H}_{k-1}^{(F)}+\sigma_{k} \underline{I}_{k} .
$$

Then, we have that both $\underline{H}_{k-1}^{(F)}$ and $\underline{K}_{k-1}^{(F)}$ are upper Hessenberg matrices and

$$
\mathbb{A} \mathbf{V}_{k}^{(F)} \underline{H}_{k-1}^{(F)}=\mathbb{B} \mathbf{V}_{k}^{(F)} \underline{K}_{k-1}^{(F)}
$$

is a Rational Krylov recurrence relation, of order $k-1$. Moreover,

$$
\text { Range }\left(\mathbf{V}_{k}^{(F)}\right)=\operatorname{Range}\left(\left(\mathbb{A}-\sigma_{k} \mathbb{B}\right)^{-1} \mathbb{B} \mathbf{V}_{k}\right) .
$$

The filtered basis $\mathbf{V}_{k}^{(F)}$ spans the Krylov space obtained by multiplying the old Krylov space $\mathbf{V}_{k}$ with $\left(\mathbb{A}-\sigma_{1} \mathbb{B}\right)^{-1} \mathbb{B}$. This operation removes the small eigenvalues from the Krylov space. Their Ritz values are usually not good approximations of eigenvalues and may lead to spurious eigenvalues. Therefore, their removal by the filter may improve the numerical behaviour of the Krylov method.

In the context of TS-CORK, the new iteration vectors take the form

$$
\mathbf{V}_{k}^{(F)}=\left[\begin{array}{c}
\left(I_{d_{\phi}+1} \otimes Q\right) \mathbf{U}^{[\phi]} \underline{Y}_{k} \\
\left(I_{d_{\psi}} \otimes L\right) \mathbf{U}^{[\psi]} \underline{Y}_{k}
\end{array}\right]
$$

where $Q$ has rank $r \leq d_{\phi}+k+1$. Since they also satisfy a recurrence relation of order $k-1$, we know that there are $Q^{(F)}, L^{(F)},\left(\mathbf{U}^{[\phi]}\right)^{(F)}$ and $\left(\mathbf{U}^{[\psi]}\right)^{(F)}$ such that

$$
\mathbf{V}_{k}^{(F)}=\left[\begin{array}{c}
\left(I_{d_{\phi}+1} \otimes Q^{(F)}\right)\left(\mathbf{U}^{[\phi]}\right)^{(F)} \\
\left(I_{d_{\psi}} \otimes L^{(F)}\right)\left(\mathbf{U}^{[\psi]}\right)^{(F)}
\end{array}\right]
$$

with rank of $Q^{(F)}$ bounded from above by $d_{\phi}+k$. It may thus happen that $Q^{(F)}$ has smaller rank than $Q$. As a result, $L^{(F)}$ may also have lower rank than $L$ since it is intimately connected to $Q^{(F)}$. Algorithm ?? shows the different steps.

For the two-sided method, we apply the algorithm on both right and left subspaces. This produces new $\mathbf{V}$ and $\mathbf{Z}$.

4. Numerical examples. In this section, we illustrate the use of the right and left Krylov spaces generated by Algorithm ?? for computing eigenvalue and eigenvector approximations. We compare the following methods:

CORK: the compact rational Krylov method applied to $\mathbf{L}_{1}$ as described in [?]. This also corresponds to Algorithm ?? applied to $\mathbf{L}_{1}$ with the standard (Euclidean) inner product denoted by $\langle\cdot, \cdot\rangle_{f}$, which does not require additional products with $A_{i}$ and $B_{i}$, since $t^{[\psi]}$ is empty. The Ritz values are obtained from (??).

T-CORK: Algorithm ?? applied to $\mathbf{L}_{2}=\mathbf{L}_{1}^{T}$. The Ritz values are again obtained from (??). The iteration vectors cannot be fully orthogonalized without additional products with $A_{i}$, $B_{i}$. We compare the convergence behaviour of Ritz values when using the inner products $\langle\cdot, \cdot\rangle_{\phi},\langle\cdot, \cdot\rangle_{\phi, \psi}$ and also the standard (expensive) inner product $\langle\cdot, \cdot\rangle_{f}$. Note that $\langle\cdot, \cdot\rangle_{\phi}$ only uses the first block, $v_{j}^{[0]}$, of iteration vector $\mathbf{v}_{j}$. 


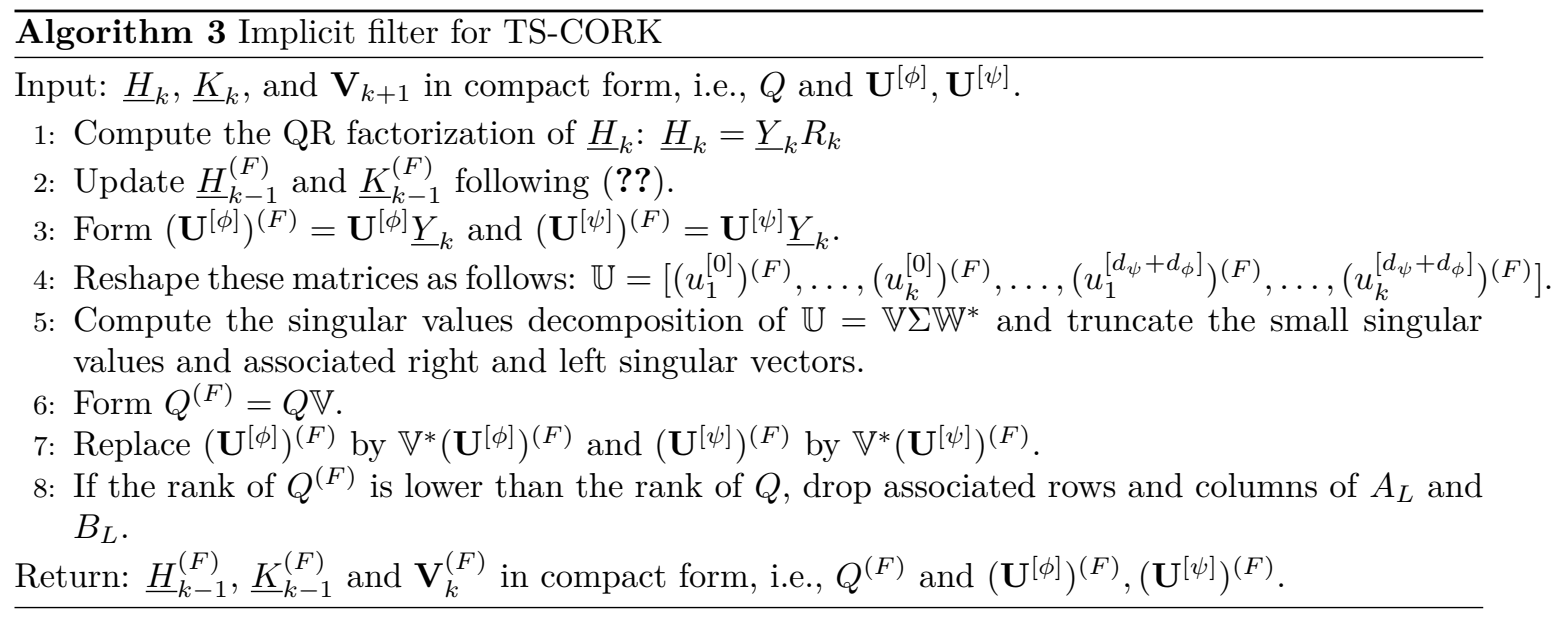

RS-CORK: Algorithm ?? applied to $\mathbf{L}_{S}$. The Ritz values are obtained from (??). Both inner products $\langle\cdot, \cdot\rangle_{\phi}$ and $\langle\cdot, \cdot\rangle_{\phi, \psi}$, are compared. In contrast to T-CORK, $\langle\cdot, \cdot\rangle_{\phi}$ uses the upper half of the iteration vectors.

LS-CORK: The same as RS-CORK, but Algorithm ?? is applied to $\mathbb{A}^{T}$ and $\mathbb{B}^{T}$ of $\mathbf{L}_{S}=\mathbb{A}-s \mathbb{B}$.

P-CORK: This is the two-sided rational Krylov method, explained in the beginning of Section ??, where $\mathbf{L}_{1}$ is projected on the left and right Krylov spaces constructed by CORK and TCORK, respectively. This method produces Ritz triplets, i.e., Ritz values and associated right and left Ritz vectors, computed from the projected pencil (??).

TS-CORK: This is the two-sided rational Krylov method applied to $\mathbf{L}_{S}$, where the right and left Krylov spaces are computed with RS-CORK and LS-CORK, respectively. This method produces Ritz triplets, i.e., Ritz values and associated right and left Ritz vectors, computed from the projected pencil (??).

As starting vector we use a vector in the low rank format

$$
\mathbf{v}_{1}=\left[\begin{array}{c}
f \otimes v_{1} \\
\mathbf{0}_{n d_{\psi}}
\end{array}\right]
$$

where $f \in \mathbb{C}^{d_{\phi}+1}, v_{1} \in \mathbb{C}^{n} \backslash\{0\}$, both with two-norm 1 , so that $Q=\left[v_{1}\right] \in \mathbb{C}^{n \times 1}$ and $\mathbf{u}^{[\phi]}=f$ and $\mathbf{u}^{[\psi]}=0$. In our experiments, we chose $f=e_{1}$.

In the numerical examples, convergence will be measured by the backward errors on the right and left eigenpair estimates, defined as the scaled residual norms

$$
R_{x}=\frac{\|A(\lambda) x\|_{2}}{\|A(\lambda)\|_{1}\|x\|_{2}}, \quad R_{y}=\frac{\left\|y^{*} A(\lambda)\right\|_{2}}{\|A(\lambda)\|_{1}\|y\|_{2}},
$$

where $x$ and $y$ correspond to the first $n$-sized block of the Ritz vectors of the linear pencil.

4.1. Gun problem. Consider the model of a radio-frequency gun cavity available from the NLEVP collection [?], known as the gun problem. This problem has the following matrix:

$$
A(\lambda)=\left(K-\lambda M+i \sqrt{\left(\lambda-\sigma_{1}^{2}\right)} W_{1}+i \sqrt{\left(\lambda-\sigma_{2}^{2}\right)} W_{2}\right) .
$$

See [?] for the details. The problem is of size $n=9956$, and $\sigma_{1}=0, \sigma_{2}=108.8774$. Since the coefficient matrices are symmetric, we can use $\mathbf{Z}=\overline{\mathbf{V}}$, i.e., a two-sided method is strictly speaking not needed to solve this problem. We use this example to compare the reliability of the CORK, T-CORK and RS-CORK methods for computing eigenvalues and right eigenvectors using (??).

We determine eigenpairs of the nonlinear eigenvalue problem, whose eigenvalues are nearest to $250^{2}$. As in [?] and [?], the eigenvalue problem is approximated by a rational eigenvalue problem expressed in a rational Newton basis of degree 21 and five shifts are used, one at $250^{2}$ and the other four around this point in the complex plane. 